\title{
PdBI U/LIRG Survey (PULS): Dense molecular gas in Arp 220 and NGC 6240
}

\author{
Kazimierz Sliwa ${ }^{1}$ and Dennis Downes ${ }^{2}$ \\ 1 Max Planck Institute for Astronomy, Königstuhl 17, 69117 Heidelberg, Germany \\ e-mail: sliwa@mpia-hd.mpg.de \\ 2 Institut de Radio Astronomie Millimetrique, Domaine Universitaire, 38406 St.-Martin d'Hères, France \\ e-mail: downes@iram.fr
}

Received 25 November 2016 / Accepted 8 April 2017

\begin{abstract}
Aims. We present new IRAM Plateau de Bure Interferometer observations of Arp 220 in $\mathrm{HCN}, \mathrm{HCO}^{+}, \mathrm{HN}^{13} \mathrm{C} J=1-0, \mathrm{C}_{2} \mathrm{H} \mathrm{N}=1-0$, $\mathrm{SiO} J=2-1$, HNCO $J_{k, k^{\prime}}=5_{0,4}-4_{0,4}, \mathrm{CH}_{3} \mathrm{CN}(6-5), \mathrm{CS} J=2-1$ and 5-4 and ${ }^{13} \mathrm{CO} J=1-0$ and 2-1 and of NGC 6240 in $\mathrm{HCN}$, $\mathrm{HCO}^{+} J=1-0$ and $\mathrm{C}_{2} \mathrm{H} \mathrm{N}=1-0$. In addition, we present Atacama Large Millimeter/submill-meter Array science verification observations of Arp 220 in CS $J=4-3$ and $\mathrm{CH}_{3} \mathrm{CN}(10-9)$. Various lines are used to analyse the physical conditions of the molecular gas including the $\left[{ }^{12} \mathrm{CO}\right] /\left[{ }^{13} \mathrm{CO}\right]$ and $\left[{ }^{12} \mathrm{CO}\right] /\left[\mathrm{C}^{18} \mathrm{O}\right]$ abundance ratios. These observations will be made available to the public. Methods. We create brightness temperature line ratio maps to present the different physical conditions across Arp 220 and NGC 6240. In addition, we use the radiative transfer code RADEX and a Monte Carlo Markov chain likelihood code to model the ${ }^{12} \mathrm{CO},{ }^{13} \mathrm{CO}$ and $\mathrm{C}^{18} \mathrm{O}$ lines of Arp 220 at $\sim 2^{\prime \prime}(\sim 700 \mathrm{pc})$ scales, where the ${ }^{12} \mathrm{CO}$ and $\mathrm{C}^{18} \mathrm{O}$ measurements were obtained from literature.

Results. Line ratios of optically thick lines such as ${ }^{12} \mathrm{CO}$ show smoothly varying ratios while the line ratios of optically thin lines such as ${ }^{13} \mathrm{CO}$ show a east-west gradient across Arp 220. The $\mathrm{HCN} / \mathrm{HCO}^{+}$line ratio differs between Arp 220 and NGC 6240, where Arp 220 has line ratios above 2 and NGC 6240 below 1 . The radiative transfer analysis solution is consistent with a warm $(\sim 40 \mathrm{~K})$, moderately dense $\left(\sim 10^{3.4} \mathrm{~cm}^{-3}\right)$ molecular gas component averaged over the two nuclei. We find $\left[{ }^{12} \mathrm{CO}\right] /\left[{ }^{13} \mathrm{CO}\right]$ and $\left[{ }^{12} \mathrm{CO}\right] /\left[\mathrm{C}^{18} \mathrm{O}\right]$ abundance ratios of $\sim 90$ for both. The abundance enhancement of $\mathrm{C}^{18} \mathrm{O}$ can be explained by stellar nucleosynthesis enrichment of the interstellar medium.
\end{abstract}

Key words. galaxies: abundances - galaxies: ISM - ISM: molecules - galaxies: starburst

\section{Introduction}

Ever since the discovery of a class of objects with extremely bright infrared emission called Ultra/Luminous Infrared Galaxies (LIRG: $L_{\mathrm{FIR}}=10^{11-12} L_{\odot}$; ULIRG: $L_{\mathrm{FIR}}>10^{12} L_{\odot}$ ) with the Infrared Astronomical Satellite (IRAS; e.g. Houck et al. 1984, 1985; Soifer et al. 1984a,b), these objects have been studied at every wavelength. High-resolution optical imaging show that most of these systems resemble merging/interacting systems (e.g. Murphy et al. 1996). Larson et al. (2016) showed that major mergers play a significant role for all sources with $L_{\mathrm{IR}} \geq 10^{11.5} L_{\odot}$. In addition, optical wavelengths show that these systems are highly dust obscured. Various star formation tracers have shown extreme star formation rates (e.g. U et al. 2012) suggesting that local U/LIRGs are great laboratories to study extreme modes of star formation. Mid-infrared observations are used to reveal the contribution of an active galactic nucleus (AGN), if present, to the far infrared luminosity (e.g. Genzel et al. 1998; Veilleux et al. 2009). Molecular gas observations via carbon monoxide $\left({ }^{12} \mathrm{CO}\right)$ have revealed rich concentrations of fuel for current and future star formation (e.g. Downes \& Solomon 1998; Bryant \& Scoville 1999; Wilson et al. 2008) and also massive, energetic molecular outflows on kiloparsec scales in several sources (e.g. Cicone et al. 2014, and references therein).

Gao \& Solomon (2004a) used HCN $J=1-0$ observations to show that there exists a tight correlation between infrared luminosity ( $L_{\mathrm{IR}}$; proxy for star formation rate) and $\mathrm{HCN} J=1-0$ (proxy for amount of dense molecular gas). This tight relation was extended to span over 7-8 orders of magnitude of $L_{\mathrm{IR}}$ by $\mathrm{Wu}$ et al. (2005). These results are interpreted to mean that $\mathrm{HCN} J=1-0$ is tracing the molecular gas component that is directly related to the star formation.

Single dish observations of the ${ }^{12} \mathrm{CO}$ isotopologue, ${ }^{13} \mathrm{CO}$ (e.g. Casoli et al. 1992; Garay et al. 1993; Aalto et al. 1991, 1995; Greve et al. 2009; Papadopoulos et al. 2012) in U/LIRGs have shown a trend of extremely weak emission relative to ${ }^{12} \mathrm{CO}$. The integrated brightness temperature line ratios of ${ }^{12} \mathrm{CO} /{ }^{13} \mathrm{CO}$ in U/LIRGs were found to be usually high $(>20)$ when compared to normal disk galaxies suggesting different interstellar medium (ISM) environments between the two classes of galaxies. High-resolution observations (e.g. Aalto et al. 1997; Casoli et al. 1999; Sliwa et al. 2012, 2013, 2014) show a similar trend with values evening exceeding 50 for some sources (Sliwa et al. 2017b). Possible explanations for the unusually high ratios include, photodissociation of ${ }^{13} \mathrm{CO}$, excitation and/or optical depth effects, or abundance variations (e.g. Casoli et al. 1992; Henkel \& Mauersberger 1993; Taniguchi et al. 1999). While photodissociation is likely ruled out as the dominant process due to the strong $\mathrm{C}^{18} \mathrm{O}$ emission (see Casoli et al. 1992), recent radiative transfer studies are showing that the $\left[{ }^{12} \mathrm{CO}\right] /\left[{ }^{13} \mathrm{CO}\right]^{1}$ abundance ratio is higher $(\geq 100)$ than what we perceive as normal (Sliwa et al. 2013, 2014, 2017a; Papadopoulos et al. 2014; Henkel et al. 2014; Tunnard et al. 2015b). In this paper,

\footnotetext{
1 Square brackets denote abundances while no brackets around ratios denote brightness temperature line ratios unless specifically stated.
} 
we model the molecular gas of the well studied ULIRG Arp 220 to determine whether it follows the $\left[{ }^{12} \mathrm{CO}\right] /\left[{ }^{13} \mathrm{CO}\right]$ trend seen in the literature.

Arp 220: Arp 220 (IRAS 15327+2340, UGC 9913, VV 540, IC 1127) is the nearest example of a ULIRG and thus well studied at every wavelength. In this advanced merger, the two nuclei are still distinguishable, separated by $1^{\prime \prime}(\sim 390$ pc; Norris 1988) and is modelled to finish merging within $6 \times 10^{8} \mathrm{yr}$ (König et al. 2012). With one of the most powerful star-forming environments in the local universe, it is a popular starburst template for dusty, star forming high redshift galaxies. Recently, Barcos-Muñoz et al. (2015) observed $33 \mathrm{GHz}$ continuum at very high-resolution $(\sim 30 \mathrm{pc})$ revealing that synchrotron radiation is dominant at this frequency. Combining the sizes measured from the $33 \mathrm{GHz}$ continuum and infrared observations, Barcos-Muñoz et al. (2015) derived very high molecular gas surface densities $\left(>2 \times 10^{5} M_{\odot} \mathrm{pc}^{-2}\right)$ and infrared surface luminosities $\left(>4 \times 10^{13} L_{\odot} \mathrm{pc}^{-2}\right)$. Although there is no clear evidence of an AGN with the $33 \mathrm{GHz}$ continuum (Barcos-Muñoz et al. 2015), Downes \& Eckart (2007) showed that the dust in the western nucleus is hot $(\sim 170 \mathrm{~K})$ and the size of the dust source is small that it implies a large surface luminosity that can only be plausible by an AGN. Wilson et al. (2014) used $691 \mathrm{GHz}$ continuum to also show that the western nucleus has a very high luminosity surface density that requires either the presence of an AGN or a "hot starburst". Lockhart et al. (2015) were able to resolve previously observed $\mathrm{H} \alpha+[\mathrm{NII}]$ emission into a bubbleshaped feature that is aligned with the western nucleus. Either an AGN or extreme star formation within the inner $\sim 100 \mathrm{pc}$ of the nuclei are the likely possibilities for the origin of this bubble. Zschaechner et al. (2016) confirm an outflow from the western nucleus by comparing their high-resolution Very Large Array (VLA) data of several molecular species to the ALMA data.

Over the last two decades, Arp 220 has been observed in high-resolution CO many times (Scoville et al. 1997, 2017; Downes \& Solomon 1998; Sakamoto et al. 1999; 2008; Downes \& Eckart 2007; Matsushita et al. 2009; Martín et al. 2011; König et al. 2012; Rangwala et al. 2015). The observations reveal a large concentration of molecular gas $\left(\sim 5 \times 10^{9} M_{\odot}\right.$; e.g. Downes \& Solomon 1998) within two compact nuclei surrounded by a diffuse kiloparsec-scale disk. Observations of rare $\mathrm{CO}$ isotopologues (i.e. ${ }^{13} \mathrm{CO}$ and $\mathrm{C}^{18} \mathrm{O}$ ) have been mainly obtained using single dish telescopes resulting in global fluxes (e.g. Greve et al. 2009; Papadopoulos et al. 2012); however, Matsushita et al. (2009) published high-resolution Submillimeter Array (SMA) ${ }^{13} \mathrm{CO}$ and $\mathrm{C}^{18} \mathrm{O} J=2-1\left(\sim 3.5^{\prime \prime}\right)$ observations showing that the two isotopologues have similar intensities where they suggest that either the lines are optically thick or there is an overabundance of $\mathrm{C}^{18} \mathrm{O}$ compared to ${ }^{13} \mathrm{CO}$.

Using Herschel Fourier Transform Spectrometer (FTS) spectra, Rangwala et al. (2011) modelled the global ${ }^{12} \mathrm{CO}$ emission from $J=1-0$ to $J=13-12$ with a 2-component molecular gas: cold, moderately dense $\left(T_{\text {kin }}=50 \mathrm{~K}\right.$ and $\left.n_{\mathrm{H}_{2}}=10^{2.8} \mathrm{~cm}^{-3}\right)$ and warm, dense $\left(T_{\text {kin }}=1350 \mathrm{~K}\right.$ and $\left.n_{\mathrm{H}_{2}}=10^{3.2} \mathrm{~cm}^{-3}\right)$ molecular gas components. The dust continuum from the FTS spectrum was also shown to be consistent with a warm dust $\left(T_{\text {dust }}=66 \mathrm{~K}\right)$ with a large optical depth $\left(\tau_{\text {dust }} \sim 5\right.$ at $100 \mu \mathrm{m}$; Rangwala et al. 2011).

Dense gas tracers such as $\mathrm{HCN}, \mathrm{HCO}^{+}, \mathrm{HNC}$ and $\mathrm{CS}$ have also been observed in Arp 220 (e.g. Aalto et al. 2009, 2015; Greve et al. 2009; Sakamoto et al. 2009; Imanishi et al. 2010; Scoville et al. 2015; Martín et al. 2011, 2016; Tunnard et al. 2015a, Barcos-Muñoz et al., in prep.). Sakamoto et al. (2009) reported PCygni profiles in the $\mathrm{HCO}^{+} J=3-2$ and $J=3-2$ suggesting a $\sim 100 \mathrm{~km} \mathrm{~s}^{-1}$ outflow originating from the inner regions of the nuclear disks. Aalto et al. (2009) found that HNC $J=3-2$ is bright and, perhaps, amplified in the western nucleus and weak in the east suggesting very different physical conditions. Martín et al. (2016) find that $\mathrm{HCN}$ and $\mathrm{HCO}^{+} J=4-3$ and $J=3-2$ are optically thick and affected by different absorption systems that can hide up to $70 \%$ of the total intrinsic emission from these lines.

NGC 6240: NGC 6240 (IRAS 16504+0228, UGC 10592, VV 617, IC 4625) is unusual among the class of LIRGs in having exceptionally strong emission in the near and mid-IR lines of molecular hydrogen. The consensus of the infrared observers is that the strong $\mathrm{H}_{2}$ lines are due to shocked gas, not star formation (Rieke et al. 1985; Depoy et al. 1986; Lester et al. 1988; Herbst et al. 1990; Elston \& Maloney 1990; van der Werf et al. 1993; Mori et al. 2014). Since the cooling time of this shocked gas is short $\left(\sim 10^{7} \mathrm{yr}\right)$, we may be seeing NGC 6240 at a privileged moment during the merger process (e.g. Sugai et al. 1997). Recent work of Meijerink et al. (2013) showed that the ${ }^{12} \mathrm{CO}$ spectral line energy distribution (SLED), obtained using the Herschel FTS, is consistent with either an X-ray dominated region (XDR) or with shocked molecular gas; however, the lack of the ionic species $\mathrm{OH}^{+}$and $\mathrm{H}_{2} \mathrm{O}^{+}$, normally found in high abundance in gas clouds near elevated $\mathrm{X}$-ray or cosmic ray fluxes, ruled out the XDR models and once again, shocks are the most likely scenario to explain the observations.

The near and mid-infrared $\mathrm{H}_{2}$ emission peaks between the two nuclei (e.g., Lester et al. 1988; Herbst et al. 1990), suggesting several possible scenarios. Two of the more recent ideas are those of Ohyama et al. (2003) and Nakanishi et al. (2005). The preferred model of Ohyama et al. (2003) is that the merger's tidal forces have channeled the molecular gas into the space between the two nuclei, where it is being shocked by a superwind from the southern nucleus, the stronger source in radio continuum, X-rays, and near- and mid-IR. Nakanishi et al. (2005) propose instead that the two nuclei have had a nearly head-on collision, after which the two supermassive black holes, the older bulge stars, and the recent starburst stars all moved onwards to the present sites of the two nuclei. Due to drag and stripping forces, however, the circumnuclear gas was left behind at the original collision site. This resembles the well-known Bullet Cluster scenario, except that collision speeds are lower, and NGC 6240's gas densities are $10^{7}$ times higher than those of the Bullet Cluster, so the gas left behind in the middle is cool molecular gas rather than hot X-ray-emitting plasma.

NGC 6240 has also been observed in high-resolution ${ }^{12}$ CO many times (Wang et al. 1991; Bryant \& Scoville 1999; Tacconi et al. 1999; Nakanishi et al. 2005; Iono et al. 2007; Wilson et al. 2008; Feruglio et al. 2013a,b). The CO emission is peaked in between the two nuclei as is observed for the warm $\mathrm{H}_{2}$ emission. Feruglio et al. (2013b) detected blueshifted CO emission between $-200 \mathrm{~km} \mathrm{~s}^{-1}$ and $-500 \mathrm{~km} \mathrm{~s}^{-1}$ peaking near the southern AGN position at the same position where an $\mathrm{H}_{2}$ outflow was found (Ohyama et al. 2000, 2003). A redshifted CO component peaks in between the two nuclei, similar to the $\mathrm{CO}$ emission at the systemic velocity, with a large velocity dispersion ( $\sim 500 \mathrm{~km} \mathrm{~s}^{-1}$ at the maximum) suggesting highly turbulent gas (Feruglio et al. 2013b).

In addition to $\mathrm{CO}$, dense gas tracers have also been observed, however, not to the extent as that for Arp 220. Nakanishi et al. (2005) observed $\mathrm{HCN}$ and $\mathrm{HCO}^{+} J=1-0$ at $2-3^{\prime \prime}$, where both lines peak in between the two nuclei, similar to $\mathrm{CO}$. Wilson et al. (2008) observed part of the $\mathrm{HCO}^{+} J=4-3$ line, where it still peaks in between the two nuclei. Tunnard et al. (2015b) 
K. Sliwa and D. Downes: Dense molecular gas in Arp 220 and NGC 6240
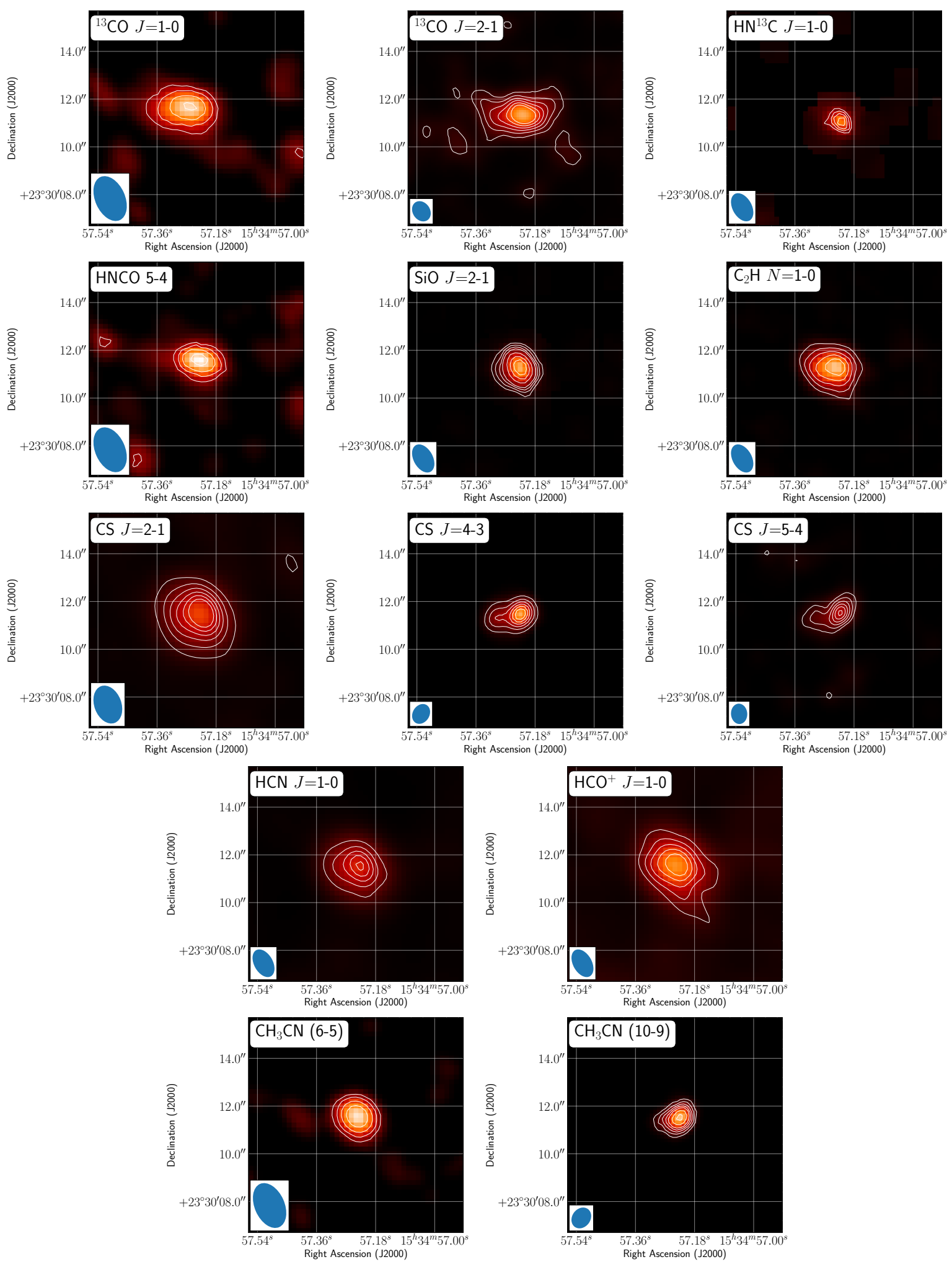

Fig. 1. Arp 220: the ellipse in the bottom left corner of each map represents the synthesized beam size. Top row: ${ }^{12} \mathrm{CO} J=1-0, J=2-1$ and $\mathrm{HN}^{13} \mathrm{C} J=1-0$ with contours corresponding to $[4,6,8,10] \times 0.45 \mathrm{Jy}_{\text {beam }}^{-1} \mathrm{~km} \mathrm{~s}^{-1},[4,8,12,16,20,24] \times 0.5 \mathrm{Jy} \mathrm{beam}^{-1} \mathrm{~km} \mathrm{~s}^{-1}$ and $[3,4,5,6] \times 0.135 \mathrm{Jy} \mathrm{beam}^{-1} \mathrm{~km} \mathrm{~s}^{-1}$, respectively. (2nd row) $\mathrm{HNCO} J_{k, k^{\prime}}=5_{0,4}-4_{0,4}, \mathrm{SiO} J=2-1$ and $\mathrm{C}_{2} \mathrm{H} N=1-0$ with contours corresponding to $[4,6,8,10] \times 0.36 \mathrm{Jy}_{\text {beam }}^{-1} \mathrm{~km} \mathrm{~s}^{-1},[4,6,8,10,15,20,25,30] \times 0.135 \mathrm{Jy} \mathrm{beam}^{-1} \mathrm{~km} \mathrm{~s}^{-1}$ and $[5,10,15,20,25] \times 0.135 \mathrm{Jy} \mathrm{beam}^{-1} \mathrm{~km} \mathrm{~s}^{-1}$, respectively. (3rd row) CS $J=2-1, J=4-3$ (ALMA) and $J=5-4$ with contours corresponding to [4, 8, 12, 16, 20, 24] $\times 0.5,1.2$ and $0.29 \mathrm{Jy}_{\text {beam }}^{-1} \mathrm{~km} \mathrm{~s}^{-1}$, respectively. (4th row) $\mathrm{HCN} J=1-0$ and $\mathrm{HCO}^{+} J=1-0$ with contours corresponding to $[5,10,15,20,25] \times 0.52$ and $0.15 \mathrm{Jy}_{\text {beam }}^{-1} \mathrm{~km} \mathrm{~s}^{-1}$, respectively. Bottom row: $\mathrm{CH}_{3} \mathrm{CN}(6-5)$ and (10-9) with contours corresponding to [4, 6, 8, 10] and [6, 12, 18, 24, 30, 40, $50] \times 0.33 \mathrm{Jy}^{-1}$ beam $^{-1} \mathrm{~km} \mathrm{~s}^{-1}$, respectively. 
Table 1. Source summary.

\begin{tabular}{lccccccc}
\hline \hline Source & $\begin{array}{c}\mathrm{R} \text { (0, 0) Position } \\
(\mathrm{J} 2000)\end{array}$ & $\begin{array}{c}\text { Dec } \\
(\mathrm{J} 2000)\end{array}$ & $\begin{array}{c}L_{\mathrm{FIR}} \\
\left(L_{\odot}\right)\end{array}$ & $\begin{array}{c}\text { Center velocity } \\
c z_{\mathrm{lsr}} \\
\left(\mathrm{km} \mathrm{s}^{-1}\right)\end{array}$ & $z_{\mathrm{lsr}}$ & $\begin{array}{c}D_{\mathrm{L}} \\
(\mathrm{Mpc})\end{array}$ & $\begin{array}{c}\text { Linear scale } \\
\left(\mathrm{pc} \operatorname{arcsec}^{-1}\right)\end{array}$ \\
\hline Arp 220 & 153457.24 & +233011.2 & $1.4 \times 10^{12}$ & 5434 & 0.018126 & 81.3 & 390 \\
NGC 6240 & 165258.89 & +022403.7 & $5.4 \times 10^{11}$ & 7340 & 0.02448 & 108 & 520 \\
\hline
\end{tabular}

Notes. [1] 9-yr WMAP parameters (Hinshaw et al. 2013): $H_{0}=69.3, \Omega_{\text {matter }}=0.28, \Omega_{\text {vacuum }}=0.72$.[2] $L_{\text {FIR }}$ reference: Sanders et al. (2003). [3] The $(0,0)$ position is the phase center of the observations.
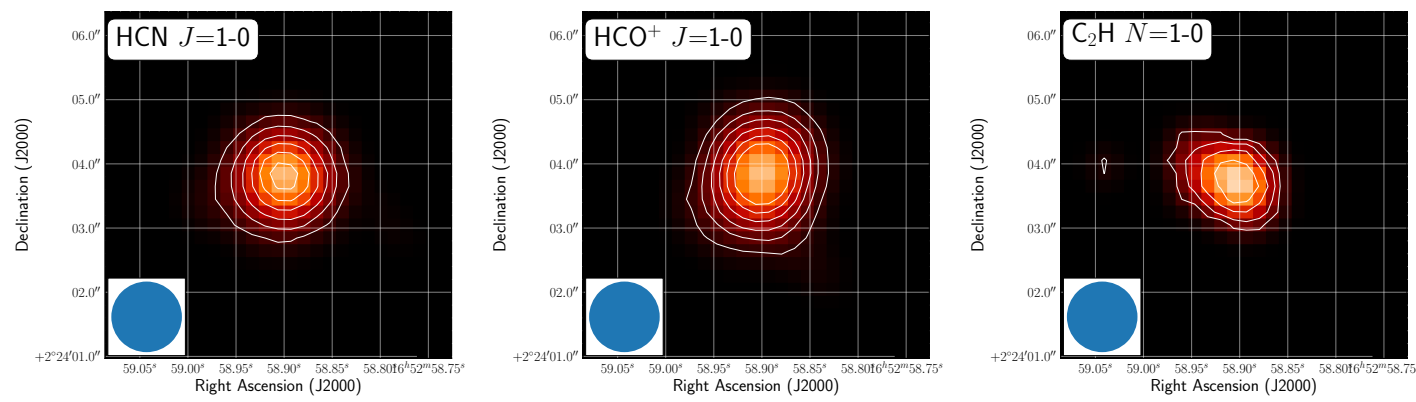

Fig. 2. NGC 6240: the ellipse in the bottom left corner of each map represents the synthesized beam size. $\mathrm{HCN} J=1-0$ and $\mathrm{HCO}^{+} J=1-0$ with contours corresponding to $[3,6,9,12,15,18] \times 0.184 \mathrm{Jy} \mathrm{beam}^{-1} \mathrm{~km} \mathrm{~s}^{-1}$ and $\mathrm{C}_{2} \mathrm{H} N=1-0$ with contours corresponding to [1, 2, 3, 4, 5] $\times$ $0.2 \mathrm{Jy} \mathrm{beam}^{-1} \mathrm{~km} \mathrm{~s}^{-1}$.

Table 2. Line Observations summary.

\begin{tabular}{|c|c|c|c|c|c|c|}
\hline Source & Line & $\begin{array}{c}v_{\text {rest }} \\
(\mathrm{GHz})\end{array}$ & $\begin{array}{l}\text { Resolution } \\
(\operatorname{arcsec})\end{array}$ & $\begin{array}{c}\mathrm{rms} \\
\left(\mathrm{mJy} \mathrm{beam}^{-1}\right)\end{array}$ & $\begin{array}{l}\text { Channel width } \\
\left(\mathrm{km} \mathrm{s}^{-1}\right)\end{array}$ & $\begin{array}{c}\text { Flux } \\
\left(\mathrm{Jy} \mathrm{km} \mathrm{s}^{-1}\right)\end{array}$ \\
\hline \multirow{13}{*}{ Arp 220} & ${ }^{13} \mathrm{CO} J=1-0$ & 110.201 & $2.0 \times 1.3$ & 1.5 & 40 & 8.0 \\
\hline & ${ }^{13} \mathrm{CO} J=2-1$ & 220.399 & $0.9 \times 0.7$ & 1.9 & 20 & 47.0 \\
\hline & $\mathrm{HCO}^{+} J=1-0$ & 89.189 & $1.3 \times 0.8$ & 0.7 & 20 & 17.8 \\
\hline & $\mathrm{HCN} J=1-0$ & 88.631 & $1.3 \times 0.8$ & 0.7 & 20 & 45.0 \\
\hline & CS $J=2-1$ & 97.981 & $1.6 \times 1.1$ & 1.3 & 20 & 17.1 \\
\hline & $\mathrm{CS} J=4-3$ & 195.954 & $0.9 \times 0.7$ & 1.4 & 20 & 61.0 \\
\hline & $\mathrm{CS} J=5-4$ & 244.936 & $0.9 \times 0.7$ & 2.5 & 40 & 40.5 \\
\hline & $\mathrm{C}_{2} \mathrm{H} N=1-0$ & $87.3 / 4$ & $1.3 \times 0.8$ & 0.8 & 20 & 8.8 \\
\hline & $\mathrm{CH}_{3} \mathrm{CN}(6-5)$ & $110.328-110.385$ & $2.0 \times 1.3$ & 1.5 & 40 & $>3.9$ \\
\hline & $\mathrm{CH}_{3} \mathrm{CN}(10-9)$ & $183.674-183.964$ & $0.9 \times 0.8$ & 2.4 & 20 & 19.7 \\
\hline & $\mathrm{HNCO}_{k_{k, k^{\prime}}}=5_{0,4}-4_{0,4}$ & 109.906 & $2.0 \times 1.3$ & 1.5 & 40 & $>2.0$ \\
\hline & $\mathrm{HN}^{13} \mathrm{CJ}=1-0$ & 87.091 & $1.3 \times 0.8$ & 0.8 & 40 & 0.7 \\
\hline & $\mathrm{SiO} J=2-1$ & 86.847 & $1.3 \times 0.8$ & 0.8 & 40 & 5.5 \\
\hline \multirow[t]{3}{*}{ NGC 6240} & $\mathrm{HCO}^{+} J=1-0$ & 89.189 & $1.1 \times 1.1$ & 1.0 & 20 & 9.9 \\
\hline & $\mathrm{HCN} J=1-0$ & 88.63 & $1.1 \times 1.1$ & 1.0 & 20 & 6.3 \\
\hline & $\mathrm{C}_{2} \mathrm{H} N=1-0$ & $87.3 / 4$ & $1.1 \times 1.1$ & 0.3 & 40 & 1.3 \\
\hline
\end{tabular}

Notes. [1] Splatalogue (http://www. splatalogue.ne/) was used to obtain frequencies. [2] $\mathrm{CH}_{3} \mathrm{CN}$ is composed of several lines that span the stated frequency range. [3] Calibration uncertainties are $\sim 5 \%$ and $\sim 10 \%$ for $3 \mathrm{~mm}$ and $1 \mathrm{~mm}$ observations, respectively.

observed the rarer ${ }^{13} \mathrm{C}$ isotopologues of $\mathrm{HCN}$ and $\mathrm{HCO}^{+}$in NGC 6240 and found line ratios $>30$.

In this paper, we present new Plateau de Bure Interferometer (PdBI) observations of $\mathrm{HCN}$ and $\mathrm{HCO}^{+} J=1-0$ and $\mathrm{C}_{2} \mathrm{H} \mathrm{N}=1-0$ for both Arp 220 and NGC 6240 (Table 1). In addition, we present new PdBI observations of ${ }^{13} \mathrm{CO} J=1-0$ and $J=2-1$, CS $J=2-1$ and $J=5-4$, HNCO $J_{k, k^{\prime}}=5_{0,4}-4_{0,4}$, $\mathrm{CH}_{3} \mathrm{CN}(6-5), \mathrm{SiO} J=2-1$, and $\mathrm{HN}^{13} \mathrm{C} J=1-0$ and Atacama Large Millimeter/submillimeter Array (ALMA) Science Verification (SV) observations of $\mathrm{CS} J=4-3$ and $\mathrm{CH}_{3} \mathrm{CN}(10-9)$ for Arp 220. These observations will be made available to the public. The paper is broken down into the following sections: in Sect. 2, we describe the observations and reduction. In Sect. 3, we present integrated brightness temperature line ratio maps to show the varying conditions of the ISM across these sources. In Sect. 4, we present a radiative transfer analysis of ${ }^{12} \mathrm{CO}$, ${ }^{13} \mathrm{CO}$ and $\mathrm{C}^{18} \mathrm{O}$ for Arp 220 at $\sim 700$ pc scales. In Sect. 5, we discuss the molecules detected in both sources, the results of the radiative transfer analysis of Arp 220 , the $\left[{ }^{12} \mathrm{CO}\right] /\left[{ }^{13} \mathrm{CO}\right]$ and $\left[{ }^{12} \mathrm{CO}\right] /\left[\mathrm{C}^{18} \mathrm{O}\right]$ abundance ratios found in Arp 220 and a comparison of the $\mathrm{HCN} / \mathrm{HCO}^{+}$line ratios. In Sect. 6, we summarize the major results. We end the paper with an appendix 
describing the release of the data online and the continuum from the observations.

\section{Observations}

\section{1. $P d B I$}

For the line-ratio investigations in this paper, we used ${ }^{12} \mathrm{CO} J=$ $1-0$ and $J=2-1$ PdBI data sets on Arp 220 and NGC 6240, that were partly data from earlier publications (for Arp 220: Downes \& Solomon 1998; Downes \& Eckart 2007; König et al. 2012; and for NGC 6240: Taconni et al. 1999). We also use the SMA observations of ${ }^{12} \mathrm{CO} J=3-2$ in Arp 220 published in Sakamoto et al. (2008) and the PdBI observations of ${ }^{12} \mathrm{CO} J=1-0$ in NGC 6240 published in Feruglio et al. (2013a).

Other, previously unpublished, data sets are, for Arp 220, ${ }^{13} \mathrm{CO} J=1-0$ at $3 \mathrm{~mm}$ and ${ }^{13} \mathrm{CO} J=2-1$ at $1.4 \mathrm{~mm}$ observed simultaneously, and also CS $J=2-1$ at $3 \mathrm{~mm}$ and CS $J=5-4$ at $1 \mathrm{~mm}$ observed simultaneously. Additional data sets included $\mathrm{HCN}, \mathrm{HN}^{13} \mathrm{C}, \mathrm{HCO}^{+} J=1-0, \mathrm{C}_{2} \mathrm{H} N=1-0$, and $\mathrm{SiO} J=2-1$ $(v=0)$ all observed simultaneously.

For most of these observations, the six $15 \mathrm{~m}$ antennas were arranged with spacings from $24 \mathrm{~m}$ to $400 \mathrm{~m}$. The longer baselines, observed in winter, had phase errors $\leq 40^{\circ}$ at $1.4 \mathrm{~mm}$, and $\leq 15^{\circ}$ at $3 \mathrm{~mm}$. Short spacings $(\leq 80 \mathrm{~m})$, observed in summer at $3 \mathrm{~mm}$, had rms phase errors $\leq 20^{\circ}$.

The SIS receiver noise plus spillover and sky noise gave typical equivalent system temperatures outside the atmosphere of $150 \mathrm{~K}$ at $3 \mathrm{~mm}(86 \mathrm{GHz})$ in the lower sideband, and 250 to $400 \mathrm{~K}$ at $1.3 \mathrm{~mm}$ in upper and lower sidebands separated by $3 \mathrm{GHz}$, with the upper band typically at 215 to $225 \mathrm{GHz}$. The spectral correlators covered $1700 \mathrm{~km} \mathrm{~s}^{-1}$ at $3 \mathrm{~mm}$ and $800 \mathrm{~km} \mathrm{~s}^{-1}$ at $1.4 \mathrm{~mm}$, with instrumental resolutions of 8 and $4 \mathrm{~km} \mathrm{~s}^{-1}$, respectively.

These raw data were then smoothed to channels of 10, 20, and $40 \mathrm{~km} \mathrm{~s}^{-1}$. The primary amplitude calibrators were $3 \mathrm{C} 273$ (a variable source, but typically $18 \mathrm{Jy}$ at $3 \mathrm{~mm}$ and $13 \mathrm{Jy}$ at $1.4 \mathrm{~mm}$, at the time of the observations), and MWC349 (a mostly nonvarying source, with 1.0 and $1.7 \mathrm{Jy}$ at 3 and $1.4 \mathrm{~mm}$ during the years of the observations). The uncertainties in the flux scales are typically $\pm 5 \%$ at $3 \mathrm{~mm}$ and $\pm 10 \%$ at $1.4 \mathrm{~mm}$.

In some of the observing epochs, the observing program monitored phases every $20 \mathrm{~min}$, at 3 and $1.4 \mathrm{~mm}$ simultaneously, with the same phase calibrators used in earlier observations of these sources (see Table 1 of Downes \& Solomon 1998). Prior to 2004 , the data processing program used the $1.4 \mathrm{~mm}$ total power to correct amplitudes and phases at 3 and $1.4 \mathrm{~mm}$ for short-term changes in atmospheric water vapor. After 2004, this was done with water-vapour monitoring receivers at $22 \mathrm{GHz}$ on each antenna. A post-observation calibration program took the $3 \mathrm{~mm}$ curve of phase versus time, scaled it to $1.4 \mathrm{~mm}$, then subtracted it from the observed $1.4 \mathrm{~mm}$ calibrator phases, and then fit the phase difference between the two receivers. All visibilities are weighted by the integration time and the inverse square of system temperature. Most maps were made with this "natural weighting" of the $u v$ data.

Some sources were observed with improved IRAM receivers in February 2008, in a $2 \times 1 \mathrm{GHz}$ spectroscopic mode that simultaneously covered the lines of $\mathrm{HCN}, \mathrm{HCO}^{+} J=1-0$ and $\mathrm{C}_{2} \mathrm{H} N=1-0$, in the newer PdBI extended configuration with antenna spacings up to $760 \mathrm{~m}$. For these later observations, typical receiver temperatures were $50 \mathrm{~K}$ at $3 \mathrm{~mm}$.
All data reductions were done with the MAPPING program in the standard IRAM GILDAS ${ }^{2}$ package.

The datacubes were converted to FITS files and imported into CASA (McMullin et al. 2007) v4.7.1 for data analysis. We created integrated intensity maps using a $1 \sigma$ cutoff for weak lines such as, for example, $\mathrm{HN}^{13} \mathrm{C}$ and a $5 \sigma$ cutoff for strong emission lines such as, for example, ${ }^{12} \mathrm{CO}, \mathrm{HCN}$ and $\mathrm{HCO}^{+}$. These integrated intensity maps are presented in Figs. 1 and 2. Table 2 presents the various molecular lines detected and their observational properties. We also present spectra in Figs. 3 and 4.

\subsection{ALMA}

Arp 220 was a target for Band 5 SV observations on 16 July 2016. The four spectral windows, of $1.875 \mathrm{GHz}$ bandwidth each, were placed on $\mathrm{H}_{2} \mathrm{O}\left(3_{13}-220\right)$, HNC $J=2-1$, CS $J=4-3$ and $\mathrm{CH}_{3} \mathrm{OH}\left(4_{31}-3_{30}\right)$. After checking for any obvious calibration flaws, we use the once phase-only self-calibrated delivered visibility dataset. We image the CS $J=4-3$ and what we have identified as $\mathrm{CH}_{3} \mathrm{CN}(10-9)$ lines. The $\mathrm{H}_{2} \mathrm{O}$ line has been presented in König et al. (2017). Using CASA v4.7.1, we create datacubes of $20 \mathrm{~km} \mathrm{~s}^{-1}$ channel widths with a natural weighting for maximum sensitivity. We created integrated intensity maps using a $2 \sigma$ cutoff (Fig. 1).

\subsection{Short-spacings flux for ${ }^{12} \mathrm{CO}$}

Greve et al. (2009) present single dish fluxes for both Arp 220 and NGC 6240. Comparing the fluxes measured for Arp 220 in Downes \& Solomon $\left(1998 ;{ }^{12} \mathrm{CO} J=1-0\right.$ and $\left.J=2-1\right)$ to that of Greve et al. (2009) shows that the PdBI maps have recovered nearly all flux ${ }^{12} \mathrm{CO} J=1-0: 410 \mathrm{Jy} \mathrm{km} \mathrm{s}^{-1}$ vs. $420 \mathrm{Jy} \mathrm{km} \mathrm{s}^{-1}$; ${ }^{12} \mathrm{CO} \mathrm{J}=2-1: 1100 \mathrm{Jy} \mathrm{km} \mathrm{s}{ }^{-1}$ vs. $1130 \mathrm{Jy} \mathrm{km} \mathrm{s}^{-1}$ ). Sakamoto et al. (2008) made comparisons to single dish observations for ${ }^{12} \mathrm{CO} J=3-2$ and concluded that $\sim 10 \%$ of the total flux is missing. Since $10 \%$ of the total flux is likely spread over the entire source, each point in Arp 220 will have insignificant missing ${ }^{12} \mathrm{CO} J=3-2$ flux when compared to the calibration uncertainty $( \pm 15 \%)$.

For NGC 6240, Feruglio et al. (2013a) made a comparison with single dish observations for ${ }^{12} \mathrm{CO} J=1-0$ and found an agreement in fluxes. The ${ }^{12} \mathrm{CO} J=2-1$ observations of Tacconi et al. (1999) are missing $\sim 30 \%$ of the total flux when compared to the flux measured in Greve et al. (2009, $1220 \mathrm{Jy} \mathrm{km} \mathrm{s}^{-1}$ vs. $1740 \mathrm{Jy} \mathrm{km} \mathrm{s}^{-1}$ ).

\section{Line ratios}

For Arp 220, we create the following integrated line brightness temperature $\left(T_{\mathrm{B}}\right)$ ratio maps for ${ }^{12} \mathrm{CO}$ and ${ }^{13} \mathrm{CO}$ :

$$
\begin{aligned}
& r_{21}=T_{\mathrm{B}}^{12} \mathrm{CO}(2-1) / T_{\mathrm{B}}^{12} \mathrm{CO}(1-0) \\
& r_{32}=T_{\mathrm{B}}^{12} \mathrm{CO}(3-2) / T_{\mathrm{B}}^{12} \mathrm{CO}(2-1) \\
& { }^{13} r_{21}=T_{\mathrm{B}}^{13} \mathrm{CO}(2-1) / T_{\mathrm{B}}^{13} \mathrm{CO}(1-0) \\
& R_{10}=T_{\mathrm{B}}^{12} \mathrm{CO}(1-0) / T_{\mathrm{B}}^{13} \mathrm{CO}(1-0) \\
& R_{21}=T_{\mathrm{B}}^{12} \mathrm{CO}(2-1) / T_{\mathrm{B}}^{13} \mathrm{CO}(2-1)
\end{aligned}
$$

We also create integrated $T_{\mathrm{B}}$ line ratio maps for molecules other than $\mathrm{CO}$ as follows:

$$
\begin{aligned}
& H_{10}=T_{\mathrm{B}}^{\mathrm{HCN}(1-0)} / T_{\mathrm{B}}^{\mathrm{HCO}^{+}(1-0)} \text { and } \\
& \mathrm{CS} / \mathrm{HNCO}=T_{\mathrm{B}}^{\mathrm{CS}(5-4)} / T_{\mathrm{B}}^{\mathrm{HNCO}(5-4)} \text { (Fig. 5). }
\end{aligned}
$$

\footnotetext{
2 http://WWW.iram.fr/IRAMFR/GILDAS
} 

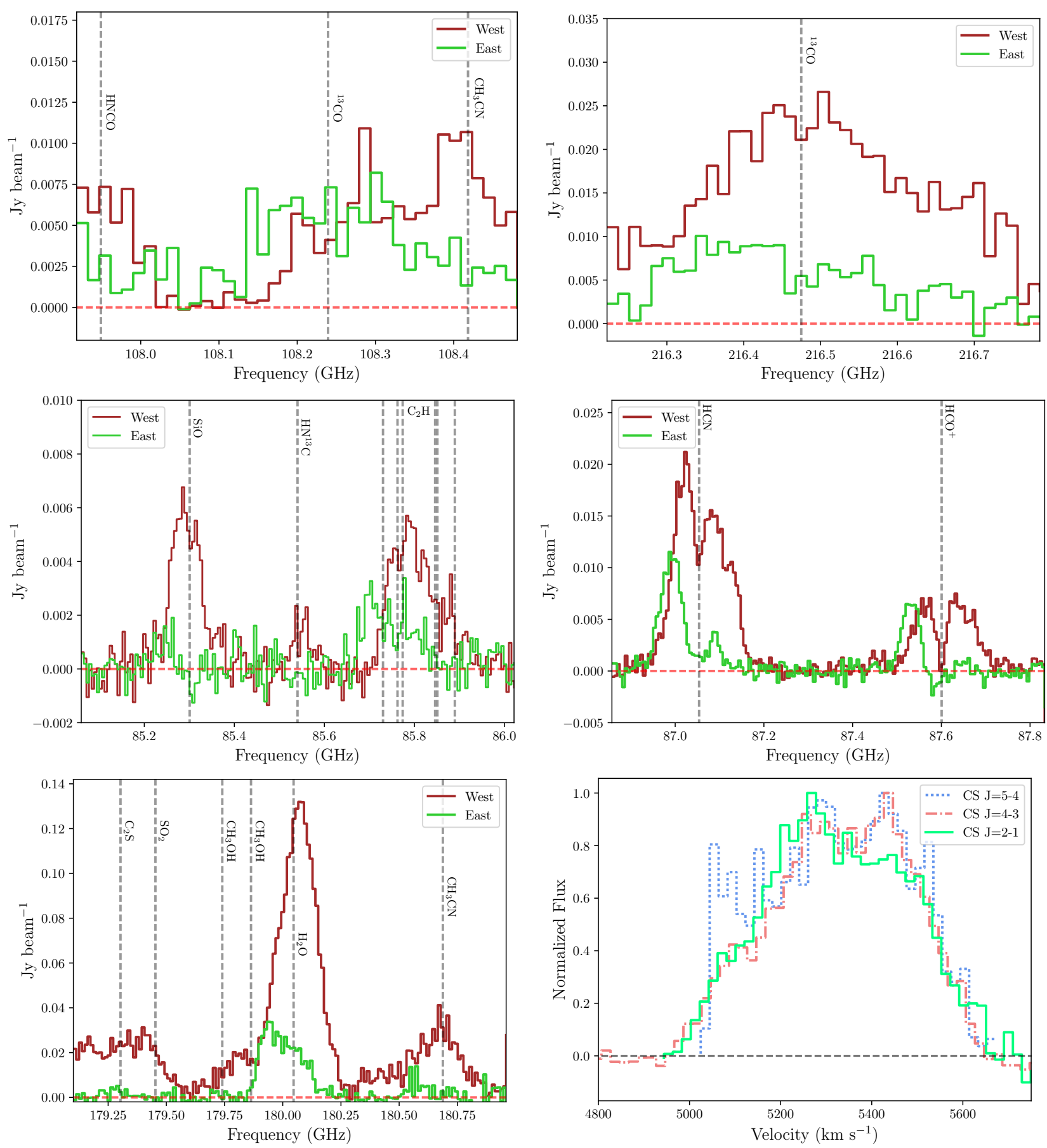

Fig. 3. Top and middle spectra averaged over a 1" diameter aperture centered at Arp 220W and Arp 220E (see Table 3 and Sect. 4) and bottom: normalized spectra of each of the CS lines averaged over a 3 " diameter aperture.

For NGC 6240, we show maps of the $T_{\mathrm{B}}$ line ratios of $r_{21}$ and $H_{10}$ (Fig. 6; with both quantities defined as for Arp 220). Table 3 gives a summary of the observed line ratios.

To match the physical scales that we analyze, we smooth the data cubes using a Gaussian kernel to match angular resolution. For Arp 220, we smooth the data to the resolution of ${ }^{13} \mathrm{CO} J=1-0$ (Table 2) and for NGC 6240, we use a compromised resolution of $1.2^{\prime \prime}$, limited by the resolution of the ${ }^{12} \mathrm{CO} J=1-0$ observations of Feruglio et al. (2013a). We applied a $5 \sigma$ cut to each map used to produce the the ratio maps.

Since ${ }^{12} \mathrm{CO}$ is believed to be optically thick, the $R_{10}$ and $R_{21}$ line ratios give a lower limit to the true $\left[{ }^{12} \mathrm{CO}\right] /\left[{ }^{13} \mathrm{CO}\right]$ abundance ratio. To illustrate this point, we start with the most general equation for the $R$ line ratios,

$$
\begin{aligned}
& R=\frac{T_{\mathrm{B}}^{12} \mathrm{CO}}{T_{\mathrm{B}}^{13} \mathrm{CO}} \\
& R=\frac{T_{\mathrm{EX}}^{12} \mathrm{CO}}{T_{\mathrm{EX}}^{13} \mathrm{CO}} \frac{\left(1-\mathrm{e}^{-\tau_{12} \mathrm{CO}}\right)}{\left(1-\mathrm{e}^{-\tau_{13} \mathrm{CO}}\right)}
\end{aligned}
$$

where $T_{\mathrm{EX}}$ is the excitation temperature, $\tau_{{ }^{12} \mathrm{CO}}$ and $\tau_{{ }^{13} \mathrm{CO}}$ are the optical depths of ${ }^{12} \mathrm{CO}$ and ${ }^{13} \mathrm{CO}$, respectively. If both ${ }^{12} \mathrm{CO}$ and ${ }^{13} \mathrm{CO}$ were to be in local thermal equilibrium (LTE) so that their 

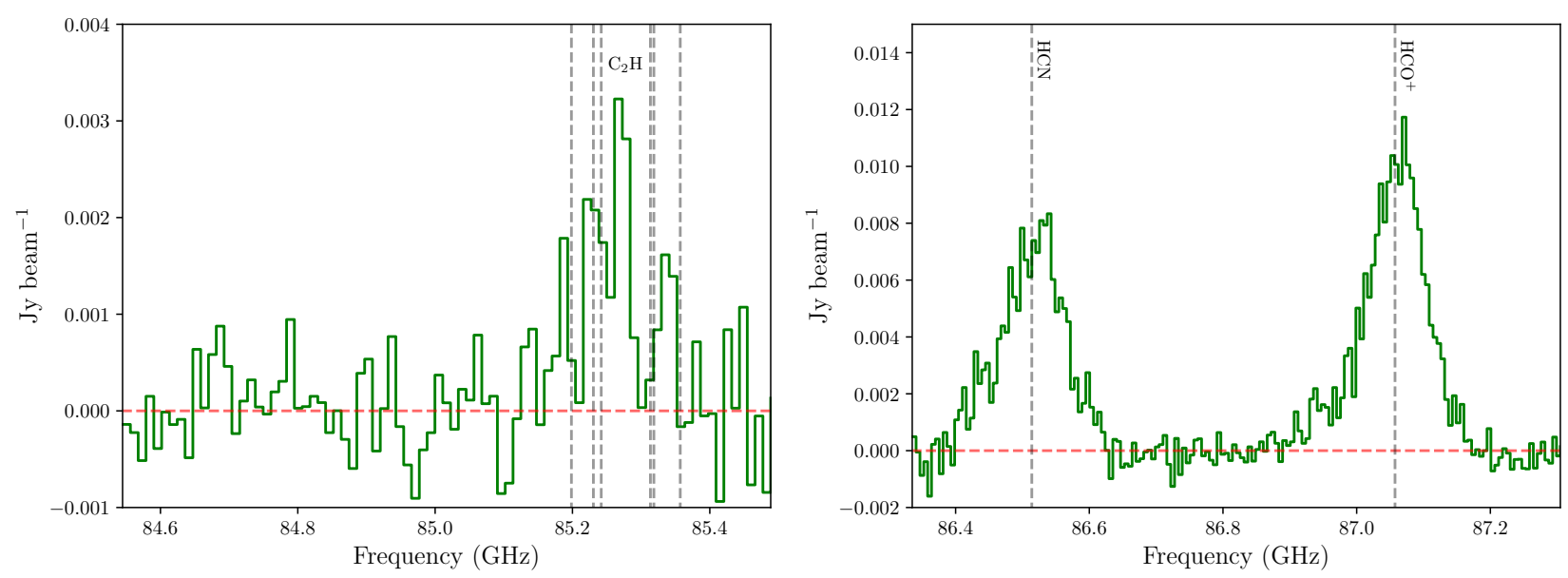

Fig. 4. Spectra averaged over a 1" diameter aperture for NGC 6240 centered on position $(0,0)$.

excitation temperatures were equal, then Eq. (1) simplifies to

$R=\frac{\left(1-\mathrm{e}^{-\tau_{12} \mathrm{CO}}\right)}{\left(1-\mathrm{e}^{-\tau_{13} \mathrm{CO}}\right)}$

In addition, if the ${ }^{12} \mathrm{CO}$ emission is sufficiently optically thick, so that $\left(1-\mathrm{e}^{-\tau_{12} \mathrm{CO}}\right) \rightarrow 1$ and if ${ }^{13} \mathrm{CO}$ is sufficiently optically thin, so that $\left(1-\mathrm{e}^{-\tau_{13} \mathrm{CO}}\right) \rightarrow \tau_{{ }^{13} \mathrm{CO}}$, then Eq. (2) can be further simplified to

$$
\begin{aligned}
R & =\frac{1}{\tau_{13} \mathrm{CO}} \\
R & =\frac{\tau_{12} \mathrm{CO}}{\tau_{13} \mathrm{CO}} \frac{1}{\tau^{12} \mathrm{CO}} \\
R & =\frac{\left[{ }^{12} \mathrm{CO}\right]}{\left.{ }^{13} \mathrm{CO}\right]} \frac{1}{\tau^{12} \mathrm{CO}}
\end{aligned}
$$

where the ratio of the optical depths of the isotopologues are equivalent to the abundance ratio ( $\tau \propto$ column density). In this case, the observed ratio $R$ of line brightness temperatures, will always be less than the true abundance, $\left[{ }^{12} \mathrm{CO}\right] /\left[{ }^{13} \mathrm{CO}\right]$, because of the attenuating factor of the ${ }^{12} \mathrm{CO}$ optical depth.

Only if ${ }^{12} \mathrm{CO}$ and ${ }^{13} \mathrm{CO}$ are both optically thin, will the observed ratio of line brightness temperatures directly trace the abundance ratio, providing that the ${ }^{12} \mathrm{CO}$ and ${ }^{13} \mathrm{CO}$ excitation temperatures are equal.

As is well-known, however, because of resonant trapping in the $\mathrm{CO}$ lines, a more realistic approach is to use one of the standard non-LTE approximations, which allows for different excitation temperatures in the different $\mathrm{CO}$ lines, and this is what we actually do (Sect. 4).

\section{Radiative transfer analysis}

To model the ${ }^{12} \mathrm{CO}$ emission (and that of the rarer $\mathrm{CO}$ isotopologues), we use the escape-probability radiative transfer program RADEX (van der Tak et al. 2007). To find the most likely RADEX solution, given the observed line strengths, and other constraints, we use the Monte Carlo Markov chain $\operatorname{code}^{3}$ of Kamenetzky et al. (2014). This code implements the nested sampling algorithm MultiNest (Feroz et al. 2009) using its Python wrapper PyMultiNest (Buchner et al. 2014) to constrain parameters. As stated in Kamenetzky et al. (2014), "The

\footnotetext{
3 https://github.com/jrka/pyradexnest
}

algorithm "nests inward" to subsequently smaller regions of high-likelihood parameter space. Unlike calculating the likelihood using a grid method, the algorithm can focus on high likelihood regions and thus estimate parameter constraints more efficiently". Model points are generated using RADEX with the following input parameters: kinetic temperature $\left(T_{\text {kin }}\right)$, column density of molecular species $\mathrm{X}$ per unit line width $\left(N_{\mathrm{X}} / \Delta \mathrm{V}\right)$ and volume density of the collision partner, molecular hydrogen $\left(n_{\mathrm{H}_{2}}\right)$. In addition, the resulting flux is allowed to scale uniformly down by an area filling factor, $\Phi_{\mathrm{A}} \leq 1$. We also implement three priors:

1. A column length to constrain the diameter of the molecular emission region. This prior assists in constraining the column and volume densities. We estimate the upper limit to the column length to be the diameter of the synthesized beam $(\sim 700 \mathrm{pc})$.

2. A dynamical mass $\left(M_{\text {dyn }}\right)$ as an upper limit to the total mass that can be contained within the column density. We use the equation from Wilson \& Scoville (1990) assuming a diameter of $\sim 700 \mathrm{pc}$ and velocity FWHMs from literature presented below.

3. An optical depth range of 0 to 100 . An optical depth below 0 implies maser emission and the upper limit of 100 is recommended by the RADEX documentation.

We refer the reader to Kamenetzky et al. (2014) for more details. We model three molecular species simultaneously: ${ }^{12} \mathrm{CO}$, ${ }^{13} \mathrm{CO}$ and $\mathrm{C}^{18} \mathrm{O}$. The ${ }^{12} \mathrm{CO}$ lines modelled are the $J=1-0,2-1$ (Downes \& Solomon 1998) and 3-2 (Sakamoto et al. 2008). We use a line ratio of ${ }^{13} \mathrm{CO} / \mathrm{C}^{18} \mathrm{O}=1$ (Matsushita et al. 2009; Greve et al. 2009) to estimate the emission of $\mathrm{C}^{18} \mathrm{O}$ at our angular resolution $\left(\sim 2^{\prime \prime}\right)$. Due to the lack of resolution elements across the ${ }^{13} \mathrm{CO}$ emission of Arp 220, we model the molecular gas at the central peak position of ${ }^{13} \mathrm{CO} J=1-0$ (see Table 2; Arp 220C) since the peak emission falls in between the two nuclei and $1^{\prime \prime}$ to each side of Arp 220C, which will place Arp 220E/W more than one resolution beam apart. We stress that Arp 220E/W are not the positions of the nuclei and are only modelled for completeness. For Arp 220W, we used only the $J=1-0$ and $2-1$ lines because the addition of the $J=3-2$ line resulted in poor SLED fits, likely indicating the presence of a second molecular gas component that cannot be fit with our data. We adopt the following linewidths: $120 \mathrm{~km} \mathrm{~s}^{-1}$ and $250 \mathrm{~km} \mathrm{~s}^{-1}$ for Arp 220W and Arp 220E measured using ALMA ${ }^{12} \mathrm{CO} J=1-0$ observations at $0.09^{\prime \prime}$ (37 pc) 

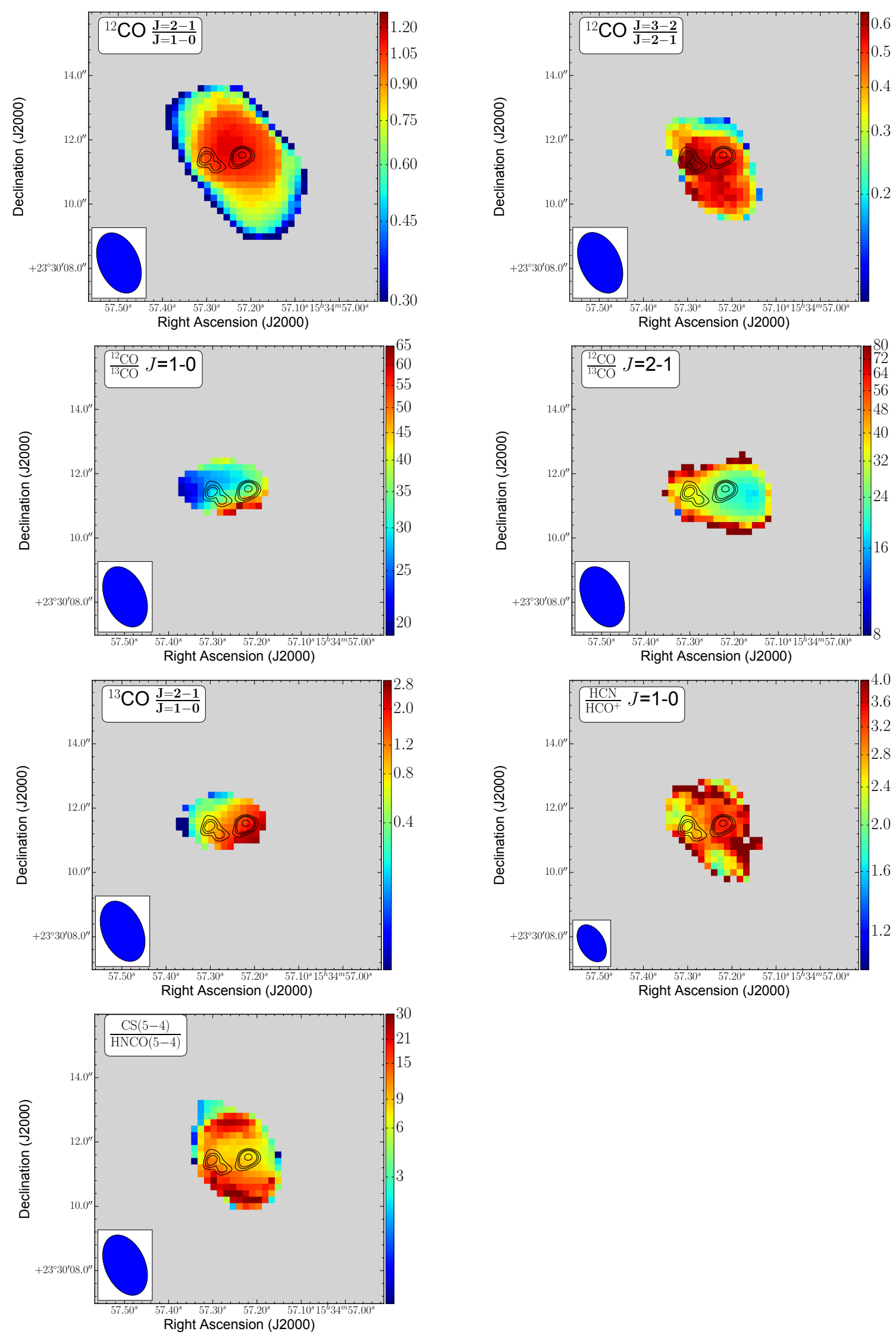

Fig. 5. Arp 220 line ratios: top $r_{21}$ and $r_{32}$, (2nd row) $R_{10}$ and $R_{21}$, (3rd row) ${ }^{13} r_{21}$ and $H_{10}$, bottom CS / HNCO. The ellipse in the bottom left corner of each map represents the synthesized beam size. The black contours represent the high-resolution ${ }^{12} \mathrm{CO} J=2-1$ emission published in Downes \& Eckart (2007) to guide the eye to the positions of the two nuclei of Arp 220. Note that the resolution of our observations do not spatially resolve the two nuclei. 

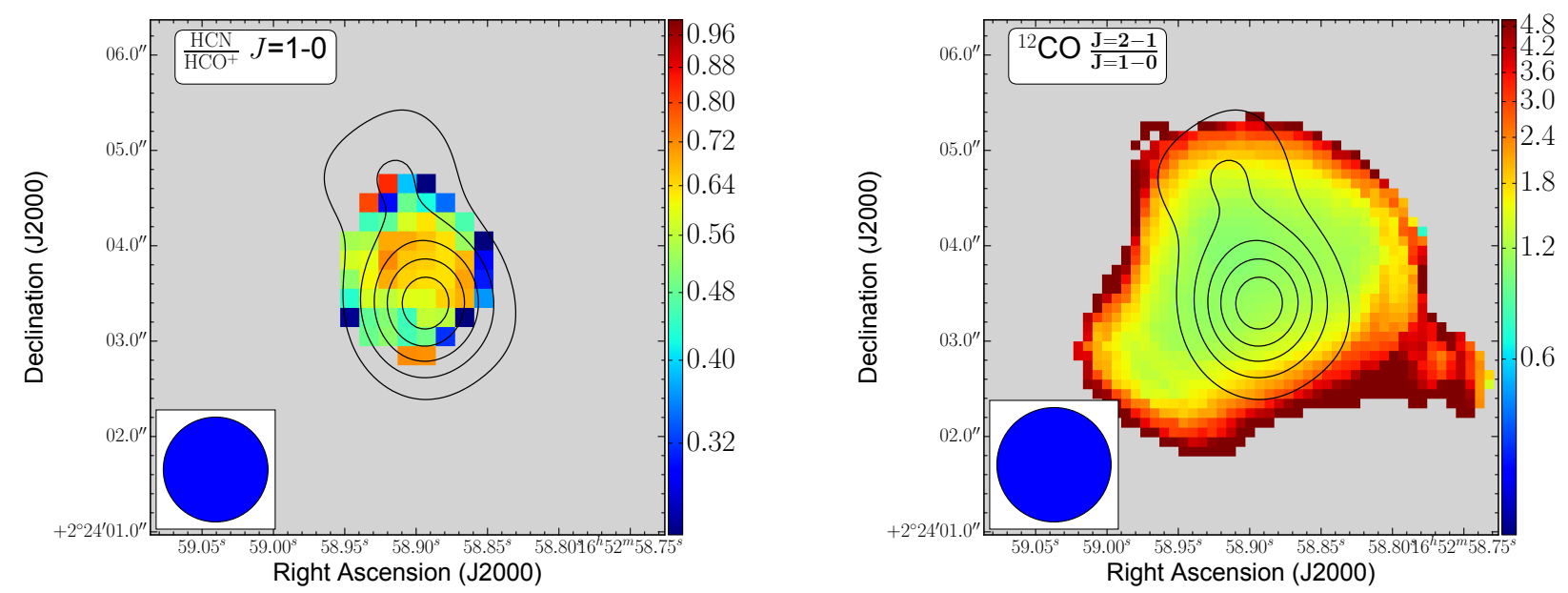

Fig. 6. NGC 6240 Line ratios: left $H_{10}$ and right $r_{21}$. The ellipse in the bottom left corner of each map represents the synthesized beam size. The black contours represent the $86 \mathrm{GHz}$ continuum emission to guide the eye to the positions of the two nuclei of NGC 6240 .

Table 3. Line ratios.

\begin{tabular}{lcccccccc}
\hline \hline \multicolumn{9}{c}{ Position } \\
& RA (J2000) & Dec (J2000) & $r_{21}$ & $r_{32}$ & $R_{10}$ & $R_{21}$ & ${ }^{13} r_{21}$ & $\mathrm{H}_{10}$ \\
\hline Arp 220E & 153457.342 & +233011.610 & $0.8 \pm 0.1$ & $0.8 \pm 0.1$ & $23 \pm 3$ & $19 \pm 2$ & $0.9 \pm 0.1$ & $2.4 \pm 0.2$ \\
Arp 220W & 153457.197 & +233011.595 & $1.1 \pm 0.1$ & $0.60 \pm 0.08$ & $38 \pm 4$ & $19 \pm 2$ & $2.3 \pm 0.3$ & $2.7 \pm 0.2$ \\
Arp 220C & 153457.258 & +233011.592 & $1.2 \pm 0.1$ & $0.60 \pm 0.08$ & $31 \pm 3$ & $26 \pm 3$ & $1.3 \pm 0.1$ & $2.5 \pm 0.2$ \\
NGC 6240 & 165258.900 & +022403.810 & $1.0 \pm 0.1$ & $\ldots$ & $\ldots$ & $\ldots$ & $\ldots$ & $0.80 \pm 0.09$ \\
\hline
\end{tabular}

Notes. The positions for Arp 220E/W do not correspond to the positions of the two nuclei. Our resolution is too poor to distinguish the nuclei spatially, therefore, the Arp 220E/W positions are $\sim 2$ " apart (greater than the synthesized beam major axis) with Arp 220C as the central position. Position error $= \pm 0.1^{\prime \prime}$.

Table 4. Radiative transfer results.

\begin{tabular}{|c|c|c|c|c|c|c|c|c|c|c|}
\hline & [1] & $\begin{array}{l}T_{\text {kin }} \\
(\mathrm{K}) \\
{[2]} \\
\end{array}$ & $\begin{array}{c}\log _{10}\left(n_{\mathrm{H}_{2}}\right) \\
\left(\mathrm{cm}^{-3}\right) \\
{[3]} \\
\end{array}$ & $\begin{array}{c}\log _{10}(\mathrm{P}) \\
\left(\mathrm{K} \mathrm{cm}^{-3}\right) \\
{[4]} \\
\end{array}$ & $\begin{array}{c}\log _{10}\left(\Phi_{\mathrm{A}}\right) \\
{[5]} \\
\end{array}$ & $\begin{array}{c}\log _{10}\left(\left\langle N_{12} \mathrm{CO}\right\rangle\right) \\
\left(\mathrm{cm}^{-2}\right) \\
{[6]} \\
\end{array}$ & $\begin{array}{c}\log _{10}\left(M_{\mathrm{H}_{2}}\right) \\
\left(M_{\odot}\right) \\
{[7]} \\
\end{array}$ & $\begin{array}{c}\alpha_{\mathrm{CO}} \\
\left(M_{\odot}\left(\mathrm{K} \mathrm{km} \mathrm{s}^{-1} \mathrm{pc}^{2}\right)^{-1}\right) \\
{[8]} \\
\end{array}$ & $\begin{array}{c}{\left[{ }^{12} \mathrm{CO}\right] /\left[{ }^{13} \mathrm{CO}\right]} \\
{[9]} \\
\end{array}$ & $\begin{array}{c}{\left[{ }^{12} \mathrm{CO}\right] /\left[\mathrm{C}^{18} \mathrm{O}\right]} \\
{[10]} \\
\end{array}$ \\
\hline \multirow[t]{4}{*}{ Arp $220 \mathrm{C}$} & Mean & 130 & 2.99 & 5.2 & -0.30 & 19.33 & 8.81 & 0.4 & 125 & 125 \\
\hline & Best Fit & 38 & 3.35 & 4.9 & -0.036 & 19.20 & 8.69 & 0.3 & 90 & 91 \\
\hline & $-1 \sigma$ Value & 37 & 2.55 & 4.9 & -0.53 & 18.97 & 8.46 & 0.2 & 58 & 56 \\
\hline & $+1 \sigma$ Value & 690 & 3.40 & 5.4 & -0.074 & 19.74 & 9.23 & 1.0 & 309 & 323 \\
\hline \multirow[t]{4}{*}{ Arp $220 \mathrm{E}$} & Mean & 240 & 2.54 & 4.9 & -0.22 & 19.04 & 8.53 & 0.4 & 93 & 93 \\
\hline & Best Fit & 34 & 3.10 & 4.6 & -0.017 & 19.24 & 8.73 & 0.6 & 159 & 159 \\
\hline & $-1 \sigma$ Value & 10 & 2.8 & 4.3 & -0.17 & 19.03 & 8.52 & 0.4 & 100 & 100 \\
\hline & $+1 \sigma$ Value & 110 & 3.4 & 4.9 & 0.0 & 19.45 & 8.94 & 1.0 & 255 & 255 \\
\hline \multirow[t]{4}{*}{ Arp 220W } & Mean & 105 & 3.78 & 5.8 & -0.32 & 19.36 & 8.85 & 0.5 & 151 & 149 \\
\hline & Best Fit & 300 & 3.2 & 5.7 & -0.7 & 19.34 & 8.83 & 0.5 & 142 & 143 \\
\hline & $-1 \sigma$ Value & 90 & 2.3 & 5.0 & -0.91 & 18.96 & 8.45 & 0.2 & 62 & 62 \\
\hline & $+1 \sigma$ Value & 1000 & 4.1 & 6.3 & -0.48 & 19.71 & 9.20 & 1.1 & 330 & 330 \\
\hline
\end{tabular}

Notes. Column [1]: statistic; Col. [2] kinetic temperature; Col. [3] volume density; Col. [4]: thermal pressure Col. [5]: filling factor; Col. [6]: ${ }^{12} \mathrm{CO}$ column density; Col. [7]: molecular gas $\left(\mathrm{H}_{2}\right)$ mass assuming $\left[{ }^{12} \mathrm{CO}\right] /\left[\mathrm{H}_{2}\right]=3 \times 10^{-4}$; Col. [8]: conversion factor $\alpha_{\mathrm{CO}}=1.36 M_{\mathrm{H}_{2}} / L_{\mathrm{CO}}$; factor of 1.36 is to account for $\mathrm{He}$; Col. [9]: $\left[{ }^{12} \mathrm{CO}\right] /\left[{ }^{13} \mathrm{CO}\right]$ abundance ratio; $\mathrm{Col}$. [10]: $\left[{ }^{12} \mathrm{CO}\right] /\left[\mathrm{C}^{18} \mathrm{O}\right]$ abundance ratio; the $\pm \sigma$ values denote the range of values within $1 \sigma$.

(Scoville et al. 2017), respectively, and $320 \mathrm{~km} \mathrm{~s}^{-1}$ for Arp 220C (Downes \& Solomon 1998) measured from PdBI ${ }^{12} \mathrm{CO} J=1-0$ observations. The mean, best fit and $\pm 1 \sigma$ results of $T_{\text {kin }}, n_{\mathrm{H}_{2}}$, $N^{12} \mathrm{CO}, \Phi_{\mathrm{A}}$, thermal pressure, molecular gas mass, CO-to- $\mathrm{H}_{2}$ conversion factor $\left(\alpha_{\mathrm{CO}}\right)$ and the relative abundances of ${ }^{13} \mathrm{CO}$ and $\mathrm{C}^{18} \mathrm{O}$ to ${ }^{12} \mathrm{CO}$ per model point are presented in Table 4 . The best fit SLED for Arp 220C is presented in Fig. 7. The (marginal) probability of a single parameter is computed by integrating over all other parameters. We present the marginal probability distributions of $T_{\text {kin }}, n_{\mathrm{H}_{2}}, N_{{ }^{12} \mathrm{CO}}$ and relative abundances of ${ }^{13} \mathrm{CO}$ and $\mathrm{C}^{18} \mathrm{O}$ as well as the $2 \mathrm{D}$ probability distribution of $\log _{10}\left(T_{\text {kin }}\right)$ versus $\log _{10}\left(n_{\mathrm{H}_{2}}\right)$ in Fig. 8.
We do not model NGC 6240 due to the lack of available high-resolution observations of ${ }^{13} \mathrm{CO}$ and the significant missing ${ }^{12} \mathrm{CO}$ flux in the existing high-resolution maps. We refer the reader to Tunnard et al. (2015b) for an extensive radiative transfer modelling of the molecular gas in NGC 6240.

\section{Discussion}

\subsection{Molecules Detected}

${ }^{13} \mathrm{CO}$ (second most common isotopologue of carbon monoxide, after $\left.{ }^{12} \mathrm{CO}\right)-$ The ${ }^{13} \mathrm{CO} J=2-1$ peak falls near the western nucleus of Arp 220 while the ${ }^{13} \mathrm{CO} J=1-0$ peak falls in between 


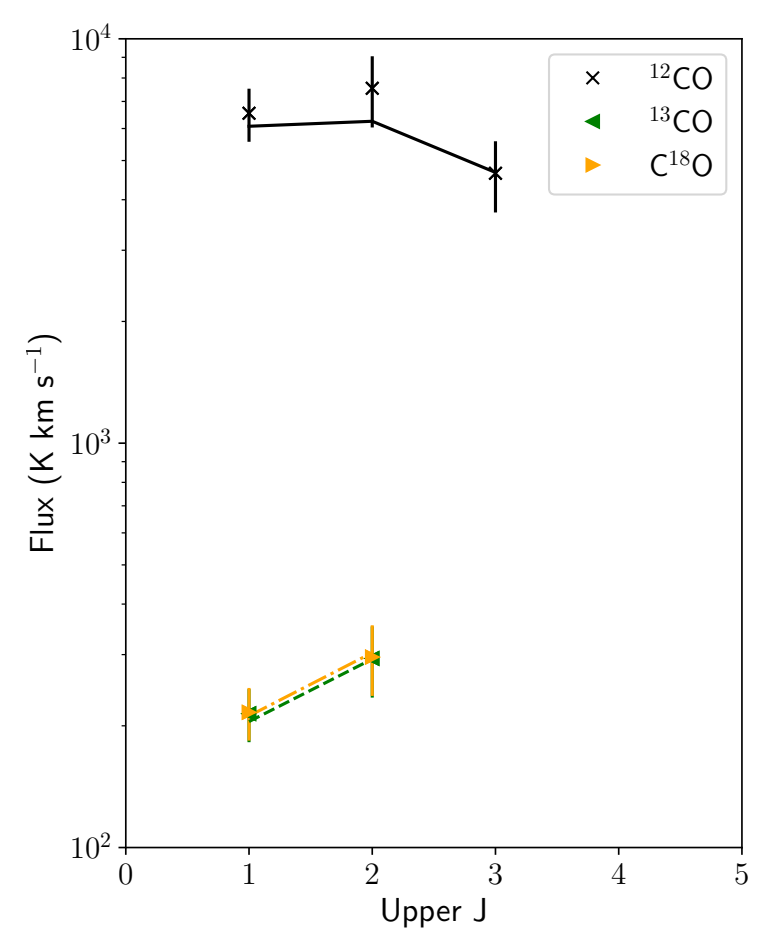

Fig. 7. Arp 220C SLED for ${ }^{12} \mathrm{CO}$ (black $\times$ ),${ }^{13} \mathrm{CO}$ (green left triangle) and $\mathrm{C}^{18} \mathrm{O}$ (orange right triangle). Solid and dashed lines represent the most probable SLED solution for each molecule.

the two nuclei. Both ${ }^{13} \mathrm{CO} J=1-0$ and $J=2-1$ have an eastwest morphology instead of a north-east south-west morphology of the ${ }^{12} \mathrm{CO}$ maps which suggests that very little ${ }^{13} \mathrm{CO}$ emission originates from the diffuse kiloparsec disk surrounding the nuclei. The ${ }^{13} \mathrm{CO}$ lines (and other optically thin tracers) are important to truly trace the physical conditions of the molecular gas as they can probe deeper into the molecular gas ensemble than the optically thick ${ }^{12} \mathrm{CO}$ (i.e. brick wall effect). This is apparent in the line ratio maps (Fig. 5) where the ${ }^{12} \mathrm{CO}$ line ratios do not vary greatly across the system while the ${ }^{13} \mathrm{CO}$ line ratios show an east-west gradient.

HCN (hydrogen cyanide) and $\mathrm{HCO}^{+}$(formyl ion) - Arp 220: the $\mathrm{HCN}$ and $\mathrm{HCO}^{+} J=1-0$ emission in Arp 220 peaks at the same position near the western nucleus which may suggest more dense gas in the west than found near the eastern nucleus. A 2D Gaussian fit of the emission shows that the size of HCN $\left(1.4^{\prime \prime} \times 1.0^{\prime \prime}\right.$; size deconvolved from the beam assuming a Gaussian structure) is more compact than $\operatorname{HCO}^{+}\left(1.9^{\prime \prime} \times 1.4^{\prime \prime}\right)$. The spectra (Fig. 3 show absorption features in both $\mathrm{HCN}$ and $\mathrm{HCO}^{+}$ similar to that seen in Martín et al. (2016) and Scoville et al. (2017). The absorption appears to have the same depth in both lines, which seem to be absorbing nearly all of the continuum. The main difference between the two lines is that HCN has more emission.

$N G C$ 6240: the integrated line flux from the $\mathrm{HCN}$ and $\mathrm{HCO}^{+} J=1-0$ is roughly half the single-dish flux measured by Greve et al. (2009). This is evidence of a larger scale HCN and $\mathrm{HCO}^{+} J=1-0$ emission that is filtered out by the interferometer. With our shortest baseline $(\sim 80 \mathrm{~m}), 50 \%$ of the flux is at scales greater than $3^{\prime \prime}(\sim 1.5 \mathrm{kpc})$. Future observations need to include shorter spacings to recover all flux.

As also observed for Arp 220, the $\mathrm{HCN}$ emission is more compact than that for $\mathrm{HCO}^{+}$which is evident in the integrated intensity maps (Fig. 2). A 2D Gaussian fit on the integrated intensity maps shows that $\mathrm{HCN}$ and $\mathrm{HCO}^{+} J=1-0$ are unresolved at our angular resolution (1.1") with an upper limit to the source size of $0.5^{\prime \prime}$ for $\mathrm{HCN}$ and $0.9^{\prime \prime} \times 0.5^{\prime \prime}$ for $\mathrm{HCO}^{+}$. The upper limits still indicate that $\mathrm{HCO}^{+} J=1-0$ is coming from a more extended component which supports the analysis of Greve et al. (2009) suggesting that $\mathrm{HCN}$ traces a denser gas phase than $\mathrm{HCO}^{+}$.

$\mathrm{C}_{2} \mathrm{H}$ (ethynyl) - the $\mathrm{C}_{2} \mathrm{H} \mathrm{N}=1-0$ transition consists of six hyperfine structure lines $(J=3 / 2-1 / 2 F=1-1,2-1,1-0$ and $J=1 / 2-1 / 2 F=1-1,0-1,1-0)$. Due to the low spectral resolution, the six lines are blended in our observations for both sources. Arp 220: the $\mathrm{C}_{2} \mathrm{H}$ emission is peaked near the western nucleus. $N G C$ 6240: the $\mathrm{C}_{2} \mathrm{H}$ emission is peaked in between the two nuclei, similar to the $\mathrm{HCN}$ and $\mathrm{HCO}^{+}$emission.

$\mathrm{SiO}$ (silicon monoxide) - the $\mathrm{SiO} J=2-1$ emission from Arp 220 has been previously observed by Tunnard et al. (2015a) at a similar spatial resolution. The total flux we measure agrees with Tunnard et al. (2015a); however, we note that there may be contamination from $\mathrm{H}^{13} \mathrm{CO}^{+} J=1-0$. Tunnard et al. (2015a) explore this possible contamination. We also do not see an absorption feature with the $\mathrm{SiO} J=2-1$ line profile in Arp 220W, however, for Arp $220 \mathrm{E}$ there is a hint of an absorption feature (Fig. 3). Since the detection in the Arp 220E is poor, it is difficult to conclude whether this is a real feature. We note that Tunnard et al. (2015a) do not see an absorption feature in $\mathrm{SiO} J=2-1$.

HNCO (isocyanic acid) and CS (carbon monosulfide): only part of the HNCO line is observed due to bandwidth constraints and may contain contamination from $\mathrm{C}^{18} \mathrm{O}$ due to the broad linewidths of Arp 220. Martín et al. (2009) observed HNCO $J_{k, k^{\prime}}=5_{0,4}-4_{0,4}$ in Arp 220 using the IRAM $30 \mathrm{~m}$ and measured a flux of $\sim 5.5 \mathrm{Jy} \mathrm{km} \mathrm{s}^{-1}$, more than twice our measured flux. The emission of $\mathrm{HNCO}$ has a horizontal elongation in the vicinity of the two nuclei with very little emission in the surrounding disk. The peak of the emission of HNCO is near the position of the western nucleus (Fig. 1); however, this may be influenced by the lack of the eastern portion of the line profile.

Greve et al. (2009) observed CS $J=2-1$ and $J=5-4$ in Arp 220. Our high-resolution observations of CS $J=2-1$ agree with the total flux within uncertainties while the CS $J=5-4$ is missing $37 \pm 12 \%$ of the total flux in the single dish map. Galametz et al. (2016) observed the CS $J=4-3$ emission from Arp 220 using APEX. The ALMA SV CS $J=4-3$ observations total flux agrees with the single dish observations. A comparison of the three CS lines (Fig. 3), shows small differences in the line profiles. The CS $J=4-3$ and $J=5-4$ have a double peak profile similar to what Greve et al. (2009) noted, while the CS $J=2-1$ line profile lacks the peak at higher velocities. The CS $J=5-4$ line also has a third peak around an observed frequency of $\sim 240.8 \mathrm{GHz}$, but this may be due to line contamination from perhaps $\mathrm{CH}_{3} \mathrm{OH}$ and/or $\mathrm{CH}_{2} \mathrm{NH}$ lines. Martín et al. (2009) observed a similar third peak in the CS $J=5-4$ line.

$\mathrm{CS}$ is a known tracer of photo-dominated regions (PDRs), where the CS is enhanced through $\mathrm{S}^{+}$chemistry (Sternberg \& Dalgarno 1995). HNCO, however, is photodissociated easily by UV photons, but is enhanced in regions with shocks (Zinchenko et al. 2000). Martín et al. (2008) proposed that the relative abundance of HNCO to CS can be used as a good diagnostic tool to distinguish between the influence of shocks and molecular clouds illuminated by UV radiation. They determined that an offset region in IC 342 (denoted by IC $342 *$ in their analysis) is dominated by shocks with a $\mathrm{CS}(5-4) / \mathrm{HNCO}(5-4)$ line ratio of $\sim 0.3$. M82 was determined to be dominated by PDRs with a $\mathrm{CS}(5-4) / \mathrm{HNCO}(5-4)$ line ratio $>64$. In the analysis of 

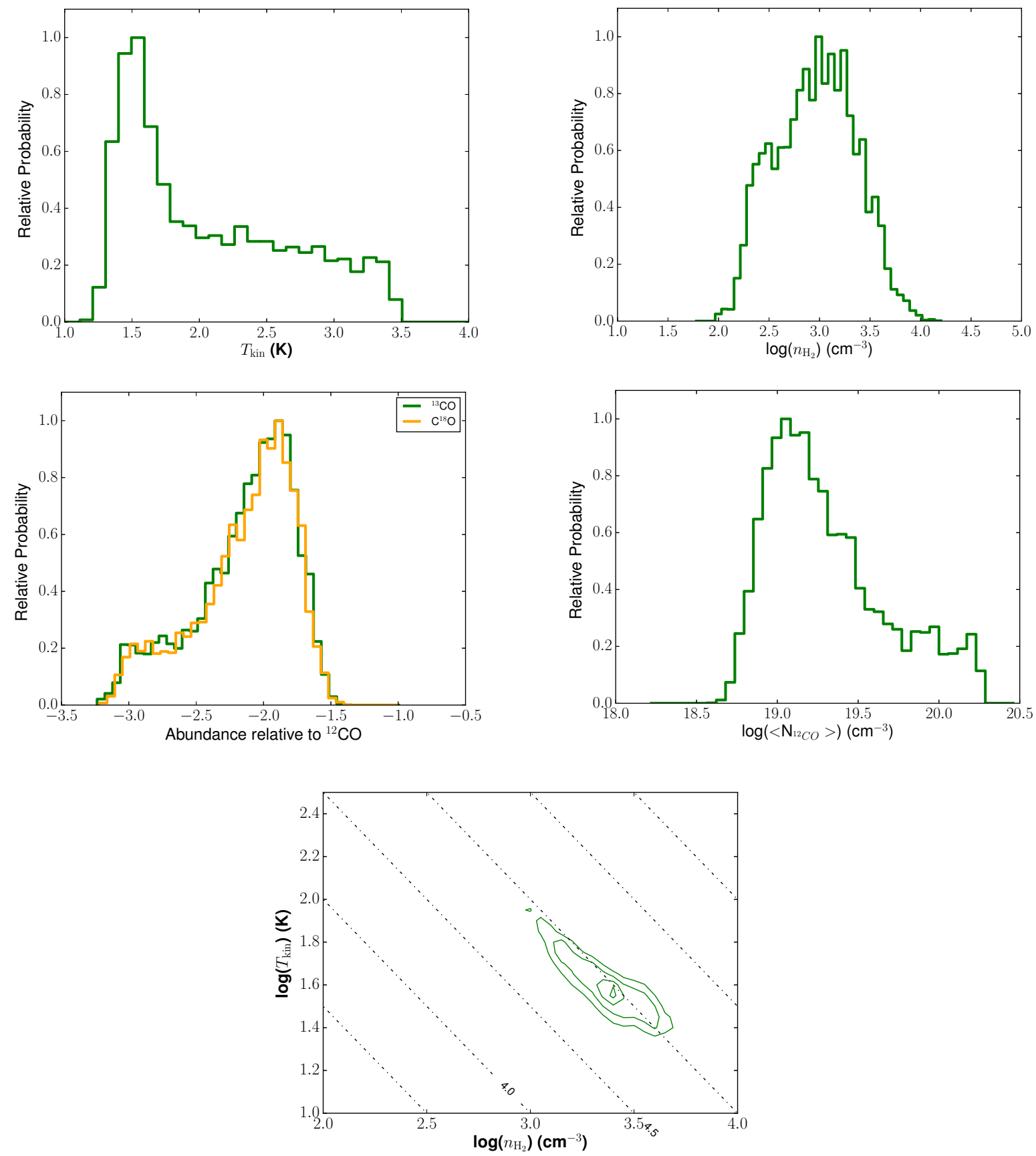

Fig. 8. Probability distributions for Arp 220C. Top left: temperature, top right: volume density, middle left: abundance of ${ }^{13} \mathrm{CO}$ and $\mathrm{C}^{18} \mathrm{O}$ relative to ${ }^{12} \mathrm{CO}$. Middle right: average column density of ${ }^{12} \mathrm{CO}$. Bottom: temperature versus volume density. Contours are $40,60,80$ and $95 \%$ of the most probable solution. Dashed lines represent $\log ($ pressure $)$.

Martin et al., Arp 220 is a composite system with contributions from PDRs and shocks. Since we only observe part of the $\mathrm{HNCO}$, our line ratios of $\mathrm{CS}(5-4) / \mathrm{HNCO}(5-4)$ (Fig. 5) is strictly an upper limit to the true line ratios; however, despite this handicap, we see significant variations of the line ratio between the region of the two nuclei $(\sim 7)$ and the kiloparsec disk $(\sim 12-30)$. Assuming both lines are optically thin and in LTE, the line ratios trace the relative abundance and this strongly suggests that the ISM in the kiloparsec disk is not strongly influenced by shocks while there is very likely a more significant contribution of shocks, likely from stellar winds, possible outflows as suggested by Sakamoto et al. (2009) and/or supernova explosions, near the two nuclei. Higher spatial resolution imaging is required to properly separate these two regions.
$H N^{13} C$ (rare isotopologue of hydrogen isocyanide) - the $\mathrm{HN}^{13} \mathrm{C}$ emission is peaked oddly below the western nucleus by $\sim 0.5 \pm 0.1^{\prime \prime}$. Nearly the entire emission is originating from the western side of Arp 220 which may be due to sensitivity since the line is weak and the eastern nucleus is the weaker emitter of the two. Wang et al. (2016) observed $\mathrm{HN}^{13} \mathrm{C} J=1-0$ and 3-2 using the IRAM $30 \mathrm{~m}$ and APEX single dish telescopes. They find that the emission is only detected in the blue component of Arp 220 (i.e. the western nucleus region) similar to what we see and our flux measurement (Table 2) agrees with theirs. Tunnard et al. (2015a) observed $\mathrm{HN}^{13} \mathrm{C} J=3-2$ in Arp 220 with the PdBI. The peaks of $\mathrm{HN}^{13} \mathrm{C} J=3-2$ are within the positions of the nuclei (both east and west; see Fig. 7 of Tunnard et al. 2015a). The flux measured by Tunnard et al. (2015a) is a 
factor of $\sim 2.5$ times higher than that of Wang et al. (2016); however, the line in the observations of Tunnard et al. (2015a) is near the bandpass edge and as noted by Wang et al. (2016), the observations may have been difficult to continuum subtract properly. If the case is that the line was poorly continuum subtracted, the peak positions of $\mathrm{HN}^{13} \mathrm{C} J=3-2$ in the PdBI map may not be due to the molecular emission but of the $1.2 \mathrm{~mm}$ continuum contamination. Using the total flux of HNC $J=1-0$ published in Greve et al. (2009) and our flux measurement for $\mathrm{HN}^{13} \mathrm{C}$ (Table 2), the line ratio of $\mathrm{HNC} / \mathrm{HN}^{13} \mathrm{C}$ is $\sim 52$. Since $\mathrm{HNC}$ is likely optically thick, this measured line ratio is a lower limit to the $\left[{ }^{12} \mathrm{C}\right] /\left[{ }^{13} \mathrm{C}\right]$ abundance.

$\mathrm{CH}_{3} \mathrm{CN}$ (methyl cyanide) - due to bandwidth limitations, only part of the $\mathrm{CH}_{3} \mathrm{CN}$ (6-5) line is observed. The $\mathrm{CH}_{3} \mathrm{CN}$ is partially blended with the ${ }^{13} \mathrm{CO} J=1-0$ line and is nearly as strong in emission as ${ }^{13} \mathrm{CO} J=1-0$. The $\mathrm{CH}_{3} \mathrm{CN}(12-11)$ line, which would be near the ${ }^{13} \mathrm{CO} J=2-1$, is not included in our bandpass; however a very small part of the $\mathrm{CH}_{3} \mathrm{CN}$ (12-11) line may be blended in the blue side of the ${ }^{13} \mathrm{CO} J=2-1$ line profile. The ALMA $\mathrm{CH}_{3} \mathrm{CN}$ (10-9) observations observed the entire line profile (Fig. 3).

Since only the emission on the blue side of the $\mathrm{CH}_{3} \mathrm{CN}(6-5)$ line is observed, it is not surprising that the emission is peaked near the western nucleus; however, when compared to the $\mathrm{CH}_{3} \mathrm{CN}$ (10-9), the peak positions agree. $\mathrm{CH}_{3} \mathrm{CN}$ is believed to be a tracer of hot cores with the relative ease of detecting the line near hot cores (e.g., Remijan et al. 2004; Purcell et al. 2006) and is a good ISM thermometer (Guesten et al. 1985) if multiple transitions are observed. Gratier et al. (2013) find that $\mathrm{CH}_{3} \mathrm{CN}$ is more abundant in UV illuminated gas and thus may be a PDR tracer. Since U/LIRGs do experience intense massive star formation, $\mathrm{CH}_{3} \mathrm{CN}$ should be relatively bright (and is in Arp 220). Observations of other transitions of $\mathrm{CH}_{3} \mathrm{CN}$ will be relatively easy in the ALMA/NOEMA era as demonstrated by the ALMA $\mathrm{SV}$ and early PdBI observations.

\subsection{Molecular gas conditions in Arp 220}

The radiative transfer modelling of the molecular gas in Arp 220 is consistent with a warm $(\sim 40 \mathrm{~K})$, moderately dense $\left(10^{3.4} \mathrm{~cm}^{-3}\right.$ molecular gas component. The best fit molecular gas density is a factor of $\sim 4$ higher than what was found by Rangwala et al. (2011) using Herschel FTS observations; however, the best fit $n_{\mathrm{H}_{2}}$ and $T_{\text {kin }}$ of the two sets of models are consistent within the $1 \sigma$ uncertainties. We note that this result should not be perceived as a lack of very dense molecular gas as been presented in Scoville et al. (2015). Our results are averaged over a $\sim 700 \mathrm{pc}$ scale which may be dominated by a less dense medium, most likely from the diffuse kiloparsec disk.

Within the $1 \sigma$ range, a warm, moderately dense gas component is favoured (Table 4 and Fig. 8). This result is very similar to other advanced mergers such as VV 114 (Sliwa et al. 2013) and NGC 2623 (Sliwa et al. 2017a) where, on average over $\sim 1 \mathrm{kpc}$ scales, a warm, moderately dense molecular gas is the best fit solution. This can be explained by feedback from massive star formation, which these mergers will experience intensely over the course of the merger process and possibly outflows. The feedback in the form of stellar winds and supernova from massive stars and the outflows that have been observed (e.g. Cicone et al. 2014) are able to push back on the molecular gas, relieving the pressure while decreasing the molecular gas volume density. Sakamoto et al. (2009) have observed a molecular PCygni profile suggesting $\sim 100 \mathrm{~km} \mathrm{~s}^{-1}$ outflows from the nuclei, which can explain our picture of a less dense medium traced by $\mathrm{CO}$. Within early/intermediate stage mergers we should see a denser molecular medium over roughly kiloparsec scales traced by $\mathrm{CO}$ and its isotopologues since the molecular gas is likely still inflowing to the central regions of the merging systems thus increasing the pressure and volume density of the molecular gas (Sliwa et al. 2017a). In Arp 220, the volume density and thus thermal pressure on $\sim$ kiloparsec scales will likely be on the rise as the two nuclei are on the verge of the final coalescence.

\section{3. $\left[{ }^{12} \mathrm{CO}\right] /\left[^{13} \mathrm{CO}\right]$ and $\left[{ }^{12} \mathrm{CO}\right] /\left[\mathrm{C}^{18} \mathrm{O}\right]$ abundance ratios in Arp 220}

The $R_{10}$ and $R_{21}$ line ratios of Arp 220 (see Table 3 ) on a position by position basis are typical for ULIRGs but abnormally high when compared with normal disk galaxies (e.g. Davis 2014) The $R_{10}$ line ratio is higher (at some positions) than other LIRGs such as Arp 299 and Arp 55 (Casoli et al. 1999; Sliwa et al. 2017a). However, these high $R_{10}$ line ratios are more common for advanced major mergers such as VV 114, IRAS 13120-5453 and NGC 2623 (Sliwa et al. 2013, 2017b,a; Saito et al. 2015). The possible explanations for the higher line ratios include optical depth effects, increased $\left[{ }^{12} \mathrm{CO}\right] /\left[{ }^{13} \mathrm{CO}\right]$ abundance ratio via some mechanism or excitation effects (e.g. Casoli et al. 1992; Henkel \& Mauersberger 1993; Taniguchi et al. 1999).

Our radiative transfer analysis allows the $\left[{ }^{12} \mathrm{CO}\right] /\left[{ }^{13} \mathrm{CO}\right]$ and $\left[{ }^{12} \mathrm{CO}\right] /\left[\mathrm{C}^{18} \mathrm{O}\right]$ abundances to vary as a free parameter. We find that for Arp 220, the most probable abundance ratio is 90-125, roughly 3 times higher than the ISM value around the Galactic center (e.g. Milam et al. 2005). In our Galaxy, the abundance ratio increases with increasing radius. If Arp 220 had no enhancements in $\left[{ }^{12} \mathrm{CO}\right] /\left[{ }^{13} \mathrm{CO}\right]$, and had a similar $\left[{ }^{12} \mathrm{CO}\right] /\left[{ }^{13} \mathrm{CO}\right]$ abundance gradient as observed in our Galaxy, then we would expect values of $\left[{ }^{12} \mathrm{CO}\right] /\left[{ }^{13} \mathrm{CO}\right] \leq 50$ since the molecular gas in Arp 220 is concentrated within $\sim 2-3 \mathrm{kpc}$. Even for NGC 6240, the most probable abundance ratio is 98 (Tunnard et al. 2015b).

Optical depth effects have been ruled out by our analysis as the best fit solutions have optical depths $>1$ for ${ }^{12} \mathrm{CO}$ and $\ll 1$ for ${ }^{13} \mathrm{CO}$.

Selective photo-dissociation was thought to be a possible mechanism to drive the unusually high $\left[{ }^{12} \mathrm{CO}\right] /\left[{ }^{13} \mathrm{CO}\right]$ abundance ratio we observe; however, this mechanism is ruled out because $\mathrm{C}^{18} \mathrm{O}$, a molecule that should be destroyed via photodissociation as fast or faster if less abundant than ${ }^{13} \mathrm{CO}$, is relatively strong in emission. The likely dominant source of the increased $\left[{ }^{12} \mathrm{CO}\right] /\left[{ }^{13} \mathrm{CO}\right]$ and $\left[{ }^{12} \mathrm{CO}\right] /\left[\mathrm{C}^{18} \mathrm{O}\right]$ abundance ratios is stellar nucleosynthesis. Short-lived $(\leq 10 \mathrm{Myr})$ massive stars produce ${ }^{12} \mathrm{C}$ and ${ }^{18} \mathrm{O}$, but ${ }^{13} \mathrm{C}$ is produced in the envelopes of long-lived low/intermediate mass during the red giant phase. If extreme starbursts have a top-heavy initial mass function, then many massive OB stars will be formed in the Arp 220 starbursts, whose supernovae will enrich the ISM, and we would expect to see increased abundances of ${ }^{12} \mathrm{C}$ and ${ }^{18} \mathrm{O}$. Matsushita et al. (2009) and Greve et al. (2009) show that the ${ }^{13} \mathrm{CO} / \mathrm{C}^{18} \mathrm{O}$ line ratio is $\sim 1$ for Arp 220 . The ${ }^{13} \mathrm{CO} / \mathrm{C}^{18} \mathrm{O}$ line ratio in spirals has been shown to be on average $\sim 6$ (Jiménez-Donaire et al. 2017), 6 times higher than for Arp 220. This strongly suggests that stellar nucleosynthesis has enriched the ISM of Arp 220. This is a similar result in other advanced mergers such as NCG 2623 and IRAS 13120-5453 (Sliwa et al. 2017b).

Fractionation of the ${ }^{12} \mathrm{C}$ and ${ }^{13} \mathrm{C}$ has also been considered as playing a role in the relative carbon isotope abundances that we find in U/LIRGs. The most important reaction to exchange carbon isotopes is

${ }^{13} \mathrm{C}^{+}+{ }^{12} \mathrm{CO} \rightleftharpoons{ }^{12} \mathrm{C}^{+}+{ }^{13} \mathrm{CO}+35 \mathrm{~K}$ 
predicted by Watson et al. (1976). The forward reaction is exothermic and in cold molecular gas can dominate isotope fractionation favouring the formation of more ${ }^{13} \mathrm{CO}$. In hotter temperatures, both the forward and reverse reactions are about equally probable and would not affect the overall abundance ratio greatly. Since the molecular gas in Arp 220 is warm $(\sim 40 \mathrm{~K})$, we can rule out fractionation as a possible contaminate in our $\left[{ }^{12} \mathrm{CO}\right] /\left[{ }^{13} \mathrm{CO}\right]$ abundance. Langer et al. (1984) showed that oxygen fractionation is very small and the oxygen isotope ratios reflect stellar nucleosynthesis processes further supporting the stellar nucleosynthesis enrichment scenario for the observed line ratios.

\section{4. $\mathrm{HCN}$ and $\mathrm{HCO}^{+}$line ratios: AGN or starburst?}

As stated in the introduction, a linear relation between $\mathrm{HCN} J=$ 1-0 and infrared luminosity was found and interpreted to be a direct correlation of dense gas and star formation (Gao \& Solomon $2004 b$ ). Within the last decade or so, the nature of HCN $J=1-0$ as a true dense gas tracer has been questioned. Studies of samples of AGN and starburst systems have found enhancements of HCN $J=1-0$ emission among the AGN systems. Recently, Privon et al. (2015) have shown that composite (i.e. AGN and star forming) and star formation dominated systems can also show a similar enhancement in $\mathrm{HCN} J=1-0$ emission. Privon et al. (2015) found that for AGN, starbursts and composite systems the $\mathrm{H}_{10}$ ratios are $1.84 \pm 0.43,0.88 \pm 0.28$ and $1.14 \pm 0.49$, respectively. This overlap of values for the different types of systems has complicated the picture of $\mathrm{HCN}$ and $\mathrm{HCO}^{+}$ emission suggesting multiple processes contributing to the line ratio differences.

Nevertheless, we compare the line ratios of Arp 220 and NGC 6240. We find that the line ratios are significantly different between the two sources. Arp 220 is consistent with AGN-like line ratios while NGC 6240 is consistent with a starburst-like ratio, similar to NGC 4039 and the Antennae Overlap region (Schirm et al. 2016). This result is quite surprising considering the fact that NGC 6240 has two well known AGNs (e.g. Komossa et al. 2003) while the presence of an AGN in Arp 220 is still debatable. The low line ratio found for NGC 6240 may reflect the different area filling factors of the two species as our observations are slightly unresolved with our beam; however, this is not the case for Arp 220 as our observations do resolve the emission and therefore, line ratios within one beam element would be filled with emission from both species. We note that our line ratio does not suggest the presence of an AGN in Arp 220 as our angular resolution does not distinguish the individual nuclei and the other possible mechanism affecting the $\mathrm{H}_{10}$ line ratios (e.g. Privon et al. 2015). Radiative transfer analyses including just these dense gas tracers are required to understand what is driving the line ratios. We also note that other effects such as infrared pumping of $\mathrm{HCN}$ and recombination of $\mathrm{HCO}^{+}$may be factors that need to be taken into consideration.

\section{Conclusions}

We present new PdBI observations of several molecular gas tracers for the two nearby major mergers Arp 220 and NGC 6240. The observations show a wealth of chemistry in Arp 220 and different conditions between the two sources. The main results are summarised as follows:

1. ${ }^{13} \mathrm{CO} J=2-1 / J=1-0$ line ratio reveal that molecular gas conditions vary across the disk of Arp 220 where the line ratio increases from east to west.
2. The best fit molecular gas conditions at the peak position of ${ }^{13} \mathrm{CO} J=1-0$ imply a warm $(40 \mathrm{~K})$, moderately dense $\left(10^{3.4} \mathrm{~cm}^{-3}\right)$ molecular gas component. This solutions differs in volume density by a factor of $\sim 4$ from the analysis of Rangwala et al. (2011) most likely because of the spatial resolution difference.

3. The $\mathrm{HCN} / \mathrm{HCO}^{+} J=1-0$ line ratio for Arp 220 is greater than 2, while for NGC 6240 the line ratios are below 1 .

4. The $\left[{ }^{12} \mathrm{CO}\right] /\left[{ }^{13} \mathrm{CO}\right]$ abundance ratio for Arp 220 is a factor of $\sim 3$ or more higher than the central value in the Milky Way, while the $\left[{ }^{12} \mathrm{CO}\right] /\left[\mathrm{C}^{18} \mathrm{O}\right]$ abundance ratio is quite low indicating the enhancement of $\mathrm{C}^{18} \mathrm{O}$ in the ISM. The likely explanation that can explain both $\left[{ }^{12} \mathrm{CO}\right] /\left[{ }^{13} \mathrm{CO}\right]$ and $\left[{ }^{12} \mathrm{CO}\right] /\left[\mathrm{C}^{18} \mathrm{O}\right]$ is an enrichment of ${ }^{12} \mathrm{C}$ and ${ }^{18} \mathrm{O}$ in the ISM via stellar nucleosynthesis. Higher-resolution and sensitive observations of ${ }^{13} \mathrm{CO}$ and $\mathrm{C}^{18} \mathrm{O}$ are needed to look for specific spatial variations as seen in IRAS 13120-5453 (Sliwa et al. 2017b).

These data, along with other sources (see Appendix B) will be made available to the public for download with the intention to use for future analyses and comparisons with new observations.

Acknowledgements. We thank the referee for their comments and suggestions that improved this manuscript. We thank K. Sakamoto for passing along the SMA ${ }^{12} \mathrm{CO} J=3-2$ observations of Arp 220. K.S. thanks Julia Kamenetzky for her MCMC code made available via github. K. S. also thanks L. BarcosMũnoz, A. K. Leroy, E. Schinnerer, F. Walter, and L. K. Zschaechner for fruitful discussions. Based on observations carried out with the IRAM Interferometer PdBI. IRAM is supported by INSU/CNRS (France), MPG (Germany) and IGN (Spain). The Submillimeter Array is a joint project between the Smithsonian Astrophysical Observatory and the Academia Sinica Institute of Astronomy and Astrophysics and is funded by the Smithsonian Institution and the Academia Sinica. This paper makes use of the following ALMA data: ADS/JAO.ALMA\#2011.0.00018.SV. ALMA is a partnership of ESO (representing its member states), NSF (USA) and NINS (Japan), together with NRC (Canada) and NSC and ASIAA (Taiwan), and KASI (Republic of Korea), in cooperation with the Republic of Chile. The Joint ALMA Observatory is operated by ESO, AUI/NRAO and NAOJ.

\section{References}

Aalto, S., Johansson, L. E. B., Booth, R. S., \& Black, J. H. 1991, A\&A, 249, 323

Aalto, S., Booth, R. S., Black, J. H., \& Johansson, L. E. B. 1995, A\&A, 300, 369 Aalto, S., Radford, S. J. E., Scoville, N. Z., \& Sargent, A. I. 1997, ApJ, 475, L107

Aalto, S., Wilner, D., Spaans, M., et al. 2009, A\&A, 493, 481

Aalto, S., Martín, S., Costagliola, F., et al. 2015, A\&A, 584, A42

Barcos-Muñoz, L., Leroy, A. K., Evans, A. S., et al. 2015, ApJ, 799, 10 Bryant, P. M., \& Scoville, N. Z. 1999, AJ, 117, 2632

Buchner, J., Georgakakis, A., Nandra, K., et al. 2014, A\&A, 564, A125

Casoli, F., Dupraz, C., \& Combes, F. 1992, A\&A, 264, 55

Casoli, F., Willaime, M.-C., Viallefond, F., \& Gerin, M. 1999, A\&A, 346, 663

Cicone, C., Maiolino, R., Sturm, E., et al. 2014, A\&A, 562, A21

Davis, T. A. 2014, MNRAS, 445, 2378

Depoy, D. L., Becklin, E. E., \& Wynn-Williams, C. G. 1986, ApJ, 307, 116

Downes, D., \& Eckart, A. 2007, A\&A, 468, L57

Downes, D., \& Solomon, P. M. 1998, ApJ, 507, 615

Elston, R., \& Maloney, P. 1990, ApJ, 357, 91

Feroz, F., Hobson, M. P., \& Bridges, M. 2009, MNRAS, 398, 1601

Feruglio, C., Fiore, F., Maiolino, R., et al. 2013a, A\&A, 549, A51

Feruglio, C., Fiore, F., Piconcelli, E., et al. 2013b, A\&A, 558, A87

Galametz, M., Zhang, Z.-Y., Immer, K., et al. 2016, MNRAS, 462, L36

Gao, Y., \& Solomon, P. M. 2004a, ApJS, 152, 63

Gao, Y., \& Solomon, P. M. 2004b, ApJ, 606, 271

Garay, G., Mardones, D., \& Mirabel, I. F. 1993, A\&A, 277, 405

Genzel, R., Lutz, D., Sturm, E., et al. 1998, ApJ, 498, 579

Gratier, P., Pety, J., Guzmán, V., et al. 2013, A\&A, 557, A101

Greve, T. R., Papadopoulos, P. P., Gao, Y., \& Radford, S. J. E. 2009, ApJ, 692, 1432 
Guesten, R., Walmsley, C. M., Ungerechts, H., \& Churchwell, E. 1985, A\&A, 142,381

Henkel, C., \& Mauersberger, R. 1993, A\&A, 274, 730

Henkel, C., Asiri, H., Ao, Y., et al. 2014, A\&A, 565, A3

Herbst, T. M., Graham, J. R., Tsutsui, K., et al. 1990, AJ, 99, 1773

Hinshaw, G., Larson, D., Komatsu, E., et al. 2013, ApJS, 208, 19

Houck, J. R., Soifer, B. T., Neugebauer, G., et al. 1984, ApJ, 278, L63

Houck, J. R., Schneider, D. P., Danielson, G. E., et al. 1985, ApJ, 290, L5

Imanishi, M., Nakanishi, K., Yamada, M., Tamura, Y., \& Kohno, K. 2010, PASJ, 62,201

Iono, D., Wilson, C. D., Takakuwa, S., et al. 2007, ApJ, 659, 283

Jiménez-Donaire, M. J., Cormier, D., Bigiel, F., et al. 2017, ApJ, 836, L29

Kamenetzky, J., Rangwala, N., Glenn, J., Maloney, P. R., \& Conley, A. 2014, ApJ, 795, 174

Komossa, S., Burwitz, V., Hasinger, G., et al. 2003, ApJ, 582, L15

König, S., García-Marín, M., Eckart, A., Downes, D., \& Scharwächter, J. 2012, ApJ, 754, 58

König, S., Martín, S., Muller, S., et al. 2017, A\&A, 602, A42

Langer, W. D., Graedel, T. E., Frerking, M. A., \& Armentrout, P. B. 1984, ApJ, 277,581

Larson, K. L., Sanders, D. B., Barnes, J. E., et al. 2016, ApJ, 825, 128

Lester, D. F., Harvey, P. M., \& Carr, J. 1988, ApJ, 329, 641

Lockhart, K. E., Kewley, L. J., Lu, J. R., et al. 2015, ApJ, 810, 149

Martín, S., Requena-Torres, M. A., Martín-Pintado, J., \& Mauersberger, R. 2008 ApJ, 678, 245

Martín, S., Martín-Pintado, J., \& Mauersberger, R. 2009, ApJ, 694, 610

Martín, S., Krips, M., Martín-Pintado, J., et al. 2011, A\&A, 527, A36

Martín, S., Aalto, S., Sakamoto, K., et al. 2016, A\&A, 590, A25

Matsushita, S., Iono, D., Petitpas, G. R., et al. 2009, ApJ, 693, 56

McMullin, J. P., Waters, B., Schiebel, D., Young, W., \& Golap, K. 2007, in Astronomical Data Analysis Software and Systems XVI, eds. R. A. Shaw, F. Hill, \& D. J. Bell, ASP Conf. Ser., 376, 127

Meijerink, R., Kristensen, L. E., Weiß, A., et al. 2013, ApJ, 762, L16

Milam, S. N., Savage, C., Brewster, M. A., Ziurys, L. M., \& Wyckoff, S. 2005 ApJ, 634, 1126

Mori, T. I., Imanishi, M., Alonso-Herrero, A., et al. 2014, PASJ, 66, 93

Murphy, Jr., T. W., Armus, L., Matthews, K., et al. 1996, AJ, 111, 1025

Nakanishi, K., Okumura, S. K., Kohno, K., Kawabe, R., \& Nakagawa, T. 2005, PASJ, 57, 575

Norris, R. P. 1988, MNRAS, 230, 345

Ohyama, Y., Yoshida, M., Takata, T., et al. 2000, PASJ, 52, 563

Ohyama, Y., Yoshida, M., \& Takata, T. 2003, AJ, 126, 2291

Papadopoulos, P. P., van der Werf, P. P., Xilouris, E. M., et al. 2012, MNRAS 426, 2601

Papadopoulos, P. P., Zhang, Z.-Y., Xilouris, E. M., et al. 2014, ApJ, 788, 153
Privon, G. C., Herrero-Illana, R., Evans, A. S., et al. 2015, ApJ, 814, 39 Purcell, C. R., Balasubramanyam, R., Burton, M. G., et al. 2006, MNRAS, 367, 553

Rangwala, N., Maloney, P. R., Glenn, J., et al. 2011, ApJ, 743, 94

Rangwala, N., Maloney, P. R., Wilson, C. D., et al. 2015, ApJ, 806, 17

Remijan, A., Sutton, E. C., Snyder, L. E., et al. 2004, ApJ, 606, 917

Rieke, G. H., Cutri, R. M., Black, J. H., et al. 1985, ApJ, 290, 116

Saito, T., Iono, D., Yun, M. S., et al. 2015, ApJ, 803, 60

Sakamoto, K., Scoville, N. Z., Yun, M. S., et al. 1999, ApJ, 514, 68

Sakamoto, K., Wang, J., Wiedner, M. C., et al. 2008, ApJ, 684, 957

Sakamoto, K., Aalto, S., Wilner, D. J., et al. 2009, ApJ, 700, L104

Sanders, D. B., Mazzarella, J. M., Kim, D.-C., Surace, J. A., \& Soifer, B. T. 2003, AJ, 126, 1607

Schirm, M. R. P., Wilson, C. D., Madden, S. C., \& Clements, D. L. 2016, ApJ, 823,87

Scoville, N., Sheth, K., Walter, F., et al. 2015, ApJ, 800, 70

Scoville, N., Murchikova, L., Walter, F., et al. 2017, ApJ, 836, 66

Scoville, N. Z., Yun, M. S., \& Bryant, P. M. 1997, ApJ, 484, 702

Sliwa, K., Wilson, C. D., Petitpas, G. R., et al. 2012, ApJ, 753, 46

Sliwa, K., Wilson, C. D., Krips, M., et al. 2013, ApJ, 777, 126

Sliwa, K., Wilson, C. D., Iono, D., Peck, A., \& Matsushita, S. 2014, ApJ, 796, L15

Sliwa, K., Wilson, C. D., Matsushita, S., et al. 2017a, ApJ, 840, 8

Sliwa, K., Wilson, C. D., Aalto, S., \& Privon, G. C. 2017b, ApJ, 840, L11

Soifer, B. T., Neugebauer, G., Helou, G., et al. 1984a, ApJ, 283, L1

Soifer, B. T., Rowan-Robinson, M., Houck, J. R., et al. 1984b, ApJ, 278, L71

Sternberg, A., \& Dalgarno, A. 1995, ApJS, 99, 565

Sugai, H., Malkan, M. A., Ward, M. J., \& McLean, I. S. 1997, ApJ, 481, 186

Tacconi, L. J., Genzel, R., Tecza, M., et al. 1999, ApJ, 524, 732

Taniguchi, Y., Ohyama, Y., \& Sanders, D. B. 1999, ApJ, 522, 214

Tunnard, R., Greve, T. R., Garcia-Burillo, S., et al. 2015a, ApJ, 800, 25

Tunnard, R., Greve, T. R., Garcia-Burillo, S., et al. 2015b, ApJ, 815, 114

U, V., Sanders, D. B., Mazzarella, J. M., et al. 2012, ApJS, 203, 9

van der Tak, F. F. S., Black, J. H., Schöier, F. L., Jansen, D. J., \& van Dishoeck, E. F. 2007, A\&A, 468, 627

van der Werf, P. P., Genzel, R., Krabbe, A., et al. 1993, ApJ, 405, 522

Veilleux, S., Rupke, D. S. N., Kim, D.-C., et al. 2009, ApJS, 182, 628

Wang, Z., Scoville, N. Z., \& Sanders, D. B. 1991, ApJ, 368, 112

Wang, J., Zhang, Z.-Y., Zhang, J., Shi, Y., \& Fang, M. 2016, MNRAS, 455, 3986

Watson, W. D., Anicich, V. G., \& Huntress, Jr., W. T. 1976, ApJ, 205, L165

Wilson, C. D., \& Scoville, N. 1990, ApJ, 363, 435

Wilson, C. D., Petitpas, G. R., Iono, D., et al. 2008, ApJS, 178, 189

Wilson, C. D., Rangwala, N., Glenn, J., et al. 2014, ApJ, 789, L36

Wu, J., Evans, II, N. J., Gao, Y., et al. 2005, ApJ, 635, L173

Zinchenko, I., Henkel, C., \& Mao, R. Q. 2000, A\&A, 361, 1079

Zschaechner, L. K., Ott, J., Walter, F., et al. 2016, ApJ, 833, 41 


\section{Appendix A: Continuum}

In this section, we present the continuum data. Table A.1 presents the continuum measurements for Arp 220 and

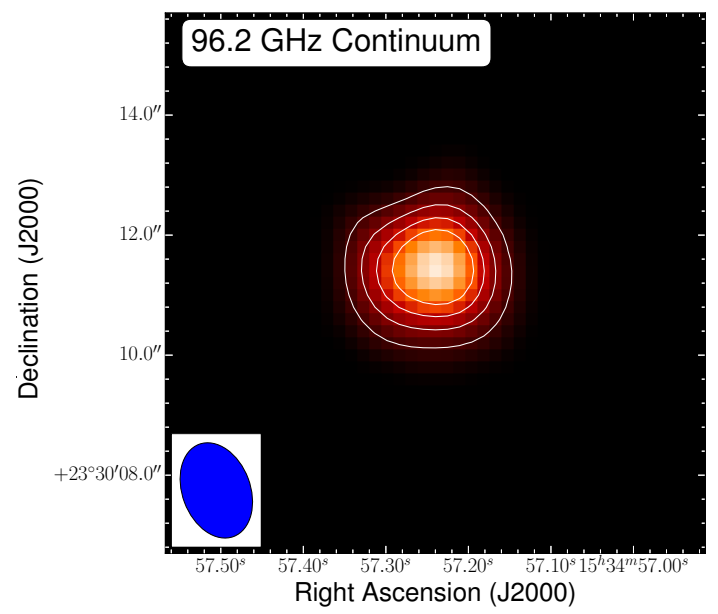

NGC 6240. Figure A.1 presents the $3 \mathrm{~mm}$ and $1 \mathrm{~mm}$ continuum for Arp 220 and $3 \mathrm{~mm}$ for NGC 6240.

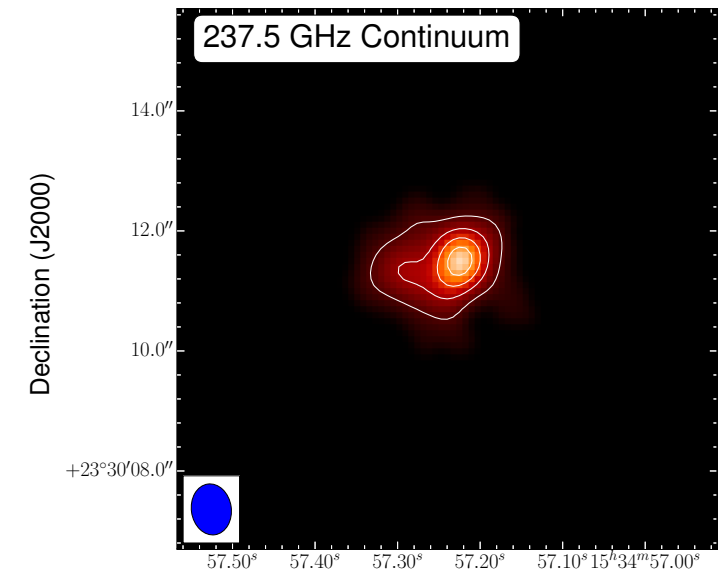

Right Ascension (J2000)

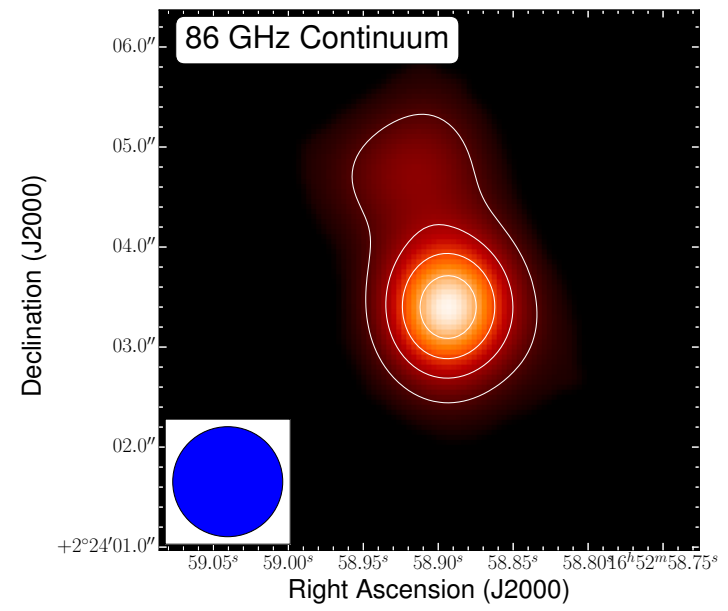

Fig. A.1. Top: continuum at $96.2 \mathrm{GHz}$ and $237.5 \mathrm{GHz}$ for Arp 220 with contours corresponding to [3, 6, 9, 12$] \times 1.0$ and $4.4 \mathrm{mJy}^{\mathrm{b}}$ beam ${ }^{-1}$, respectively. Bottom: continuum at $86 \mathrm{GHz}$ for NGC 6240 with contours corresponding to $[3,6,9,12] \times 0.32 \mathrm{mJy} \mathrm{beam}^{-1}$.

Table A.1. Continuum emission.

\begin{tabular}{|c|c|c|c|c|c|c|}
\hline \multirow[b]{2}{*}{ Source } & \multicolumn{2}{|c|}{ Continuum position } & \multirow[b]{2}{*}{$\begin{array}{c}\text { Frequency } \\
(\mathrm{GHz})\end{array}$} & \multicolumn{2}{|c|}{ Flux density } & \multirow{2}{*}{$\begin{array}{c}\text { Resolution } \\
\text { (arcsec) }\end{array}$} \\
\hline & $\begin{array}{c}\text { RA } \\
(\mathrm{J} 2000)\end{array}$ & $\begin{array}{c}\text { Dec } \\
(\mathrm{J} 2000)\end{array}$ & & $\begin{array}{c}\overline{\text { peak }} \\
\left(\mathrm{mJy} \mathrm{beam}^{-1}\right)\end{array}$ & $\begin{array}{l}\text { total } \\
(\mathrm{mJy})\end{array}$ & \\
\hline Arp 220 & 153457.24 & +233011.2 & 87.0 & 23.0 & 31.0 & $1.3 \times 0.8$ \\
\hline & & & 96.2 & 17.8 & 26.0 & $1.6 \times 1.1$ \\
\hline & & & 105.1 & 22.0 & 25.0 & $2.0 \times 1.3$ \\
\hline & & & 113.2 & 30.0 & 41.0 & $2.0 \times 1.3$ \\
\hline & & & 219.5 & 42.0 & 99.0 & $0.9 \times 0.7$ \\
\hline & & & 237.5 & 62.0 & 150.0 & $0.9 \times 0.7$ \\
\hline NGC 6240S & 165258.893 & +022403.4 & 86.5 & 4.6 & 5.1 & $1.1 \times 1.1$ \\
\hline NGC $6240 \mathrm{~N}$ & 165258.918 & +022404.8 & 86.5 & 1.7 & 1.8 & $1.1 \times 1.1$ \\
\hline NGC 6240 & & & 86.5 & 1.7 & 6.9 & $1.1 \times 1.1$ \\
\hline
\end{tabular}

Notes. Errors: position $= \pm 0^{\prime \prime} .1,3 \mathrm{~mm}$ fluxes $= \pm 5 \%, 1 \mathrm{~mm}$ fluxes $= \pm 10 \%$. 
Table B.1. Sources observed.

\begin{tabular}{cl}
\hline \hline Source & Lines \\
\hline IRAS 00057+4021 & $\mathrm{HCN}(1-0),{ }^{12} \mathrm{CO}(1-0),{ }^{12} \mathrm{CO}(2-1)$ \\
VII Zw 31 & $\mathrm{HCN}(1-0),{ }^{12} \mathrm{CO}(1-0),{ }^{12} \mathrm{CO}(2-1),{ }^{13} \mathrm{CO}(1-0),{ }^{13} \mathrm{CO}(2-1)$ \\
UGC 5101 & $\mathrm{HCN}(1-0),{ }^{12} \mathrm{CO}(1-0),{ }^{12} \mathrm{CO}(2-1)$ \\
IRAS 10565+2448 & $\mathrm{HCN}(1-0),{ }^{12} \mathrm{CO}(2-1)$ \\
Mrk 231 & $\mathrm{HCN}(1-0), \mathrm{HCO}^{+}(1-0),{ }^{12} \mathrm{CO}(2-1)$ \\
Arp 193 & $\mathrm{HCN}(1-0),{ }^{12} \mathrm{CO}(1-0),{ }^{12} \mathrm{CO}(2-1),{ }^{13} \mathrm{CO}(2-1)$ \\
Mrk 273 & $\mathrm{HCN}(1-0),{ }^{12} \mathrm{CO}(1-0),{ }^{12} \mathrm{CO}(2-1),{ }^{13} \mathrm{CO}(2-1)$ \\
IRAS 17208-0014 & $\mathrm{HCN}(1-0),{ }^{12} \mathrm{CO}(1-0),{ }^{12} \mathrm{CO}(2-1)$ \\
IRAS 23365+3604 & $\mathrm{HCN}(1-0),{ }^{12} \mathrm{CO}(1-0),{ }^{12} \mathrm{CO}(2-1)$ \\
\hline
\end{tabular}

\section{Appendix B: Release of data}

The PdBI data presented in this paper will be released via the IRAM Large Program Archive and NASA's Extragalactic Database apart of the PdBI U/LIRG Survey (PULS). Several other U/LIRGs were also observed (see Table B.1) and will be released as well. The data taken in between 2003-2008 and for most sources, the PdBI was setup to observe HCN $J=1-0$ at $3 \mathrm{~mm}$ and ${ }^{13} \mathrm{CO} J=2-1$ at $1.4 \mathrm{~mm}$, simultaneously. In the weaker sources, ${ }^{12} \mathrm{CO} J=2-1$ was observed instead of ${ }^{13} \mathrm{CO} J=2-1$. The six $15 \mathrm{~m}$ antennas were arranged with spacings from $24 \mathrm{~m}$ to $400 \mathrm{~m}$. The long baselines, observed in winter, had rms phase errors $\leq 40^{\circ}$ at $1.4 \mathrm{~mm}$, and $\leq 15^{\circ}$ at $3 \mathrm{~mm}$. Short spacings $(\leq 80 \mathrm{~m})$, observed in summer at $3 \mathrm{~mm}$, had rms phase errors $\leq 20^{\circ}$.

A paper on the analysis of Arp 193 and VII Zw 31 is forth coming (Sliwa \& Downes, in prep.). 